\title{
Determination of the cross sections for the production of fragments from relativistic nucleus-nucleus interactions. II. Parametric fits
}

\author{
J. R. Cummings, ${ }^{(1)}$ * W. R. Binns, ${ }^{(2)}$ T. L. Garrard, ${ }^{(3)}$ M. H. Israel, ${ }^{(2)}$ J. Klarmann, ${ }^{(2)}$ E. C. Stone, ${ }^{(3)}$ and \\ C. J. Waddington ${ }^{(1)}$ \\ ${ }^{(1)}$ School of Physics and Astronomy, University of Minnesota, Minneapolis, Minnesota 55455 \\ ${ }^{(2)}$ Department of Physics and the McDonnell Center for the Space Sciences, Washington University, \\ St. Louis, Missouri 63130 \\ ${ }^{(3)}$ George W. Downs Laboratory, California Institute of Technology, Pasadena, California 91125 \\ (Received 21 September 1989; revised manuscript received 3 May 1990)
}

\begin{abstract}
Measurements of the partial charge-changing cross sections for the fragmentation of relativistic iron, lanthanum, holmium, and gold nuclei of several different energies incident on targets of polyethylene, carbon, aluminum, and copper have been reported in an accompanying paper. This paper describes the systematics of the variations of these cross sections with energy, projectile, target, and fragment. We have been able to generate a seven-parameter global fit to 795 measured cross sections for the heavy targets which fits the data with a standard deviation of $7 \%$. We have also generated a similar global fit to 303 measured cross sections for a hydrogen target which fits the data with a standard deviation of $10 \%$. These representations imply that the hypothesis of limiting fragmentation is only accurate to some $20-30 \%$. Weak factorization can apply, but fits that are marginally better, and more physically plausible, can be obtained without factorization. We have identified, and discussed, a number of caveats to the applicability of these fits outside, and inside, the range of energies and masses covered. Excessively large cross sections for the loss of a single proton from the projectile nuclei suggest electromagnetic dissociation. The cross sections for fragments that experience large charge changes appear to become independent of the size of the charge change. Very heavy projectiles have a significant probability of experiencing fission.
\end{abstract}

\section{INTRODUCTION}

In a comparison paper we reported ${ }^{1}$ the experimental results of an analysis of heavy-nuclei interactions detected in an inclusive experiment conducted at the Lawrence Berkeley Laboratory's (LBL) Bevalac particle accelerator. Here we report on our analysis of the systematics of the partial cross sections for the production of heavy fragments and describe a global fit that allows the prediction of those cross sections that are not measured directly. This analysis provides a procedure to calculate the cross sections for the production of heavy fragments from charge-changing interactions between heavy-projectile nuclei with energies of at least several hundred $\mathrm{MeV} /$ nucleon and various target nuclei. These cross sections can be applied to the problem of the propagation of cosmic ray nuclei through the interstellar medium, and provide data on the nuclear physics involved in peripheral collisions of heavy nuclei. These cross sections can also be used in the calibration and correction of data from instruments that detect cosmic ray nuclei. In particular these results are relevant to our earlier experiment $^{2}$ on the High-Energy Astronomy Observatory (HEAO 3) Heavy-Nuclei Experiment (HNE), which was designed to detect the very rare ultraheavy (UH) nuclei, those with $Z \geq 30$, present in the cosmic radiation.

The abundances of these UH nuclei are important in distinguishing models of nucleosynthesis and acceleration in the cosmic ray source region. The dominant process of nucleosynthesis for the $\mathrm{UH}$ nuclides is neutron capture, via either the $s$ or $r$ process. The elemental distributions that result from these two processes are distinctly different. The source population of cosmic rays is presumably the result of some mixture of the products of these processes, modified by any preferential acceleration mechanisms that may be acting. ${ }^{3,4}$ The relative abundances of the UH elements that are detected at Earth are also modified by nuclear interactions that occur during propagation through the interstellar medium, as well as by interactions that occur in the material of the detectors themselves. In order to investigate either the source population or the propagation problem we need to know both the total charge-changing cross sections, which determine the rate of destruction of a given element, and the partial cross sections for the production of lighter secondary nuclei from heavier primary nuclei. It is necessary to know these cross sections for both hydrogen and heavy targets, since hydrogen is the main component of the interstellar medium and the detectors contain heavy materials. To solve the problem fully, we should also know the isotopic composition of the fragments that are produced in the hydrogen interactions, since the cosmic ray nuclei travel for sufficient time in the interstellar medium for most radioactive decays to occur between interactions, whereas decays will not be important in the material in the detectors.

An important empirical result that we found from an 
earlier exposure ${ }^{5}$ was that the partial cross sections for the production of fragments with a particular charge change of $\Delta Z$, varied either as a power of $\Delta Z$ in the case of heavy targets, or as an exponential of $\Delta Z$ in the case of a hydrogen target. The coefficients and the exponents in both cases varied with either or both energy and projectile mass. However, we could not separate the two dependences because all the projectiles used in that exposure were accelerated in partially stripped states to the maximum rigidity of the Bevalac, and thus had energies that depended on their masses. Hence, we decided to perform additional experiments designed to investigate the energy dependence of the $\Delta Z$ dependence of the partial cross sections separately from projectile mass dependence. The companion paper ${ }^{1}$ is a report on these results, obtained at as many as six different energies for a given projectile-target combination. We have also previously reported $^{6}$ on the small charge changes observed during these runs, concentrating our attention in that paper on the mass and energy changes observed in the lowerenergy runs and on the effects when $\Delta Z=+1$ to -3 . Here we obtain general relationships for the cross sections, for all energies, and for $\Delta Z$ down to -20 , in terms of the various physical parameters of the projectile and target nuclei.

We have, from the combined data sets, values for the cross sections for charge change of five projectiles $(\mathrm{Kr}$, $\mathrm{Xe}, \mathrm{La}, \mathrm{Ho}$, and $\mathrm{Au}$ ) on three nonhydrogenous targets $(\mathrm{C}$, $\mathrm{Al}$, and $\mathrm{Cu}$ ) at several energies ranging between 489 and $1415 \mathrm{MeV} /$ nucleon, for a total of 42 combinations of projectile and energy. For hydrogen targets we have deduced cross sections for 16 projectile-energy combinations using $\mathrm{Xe}, \mathrm{La}, \mathrm{Ho}$, and $\mathrm{Au}$ by comparing the values in polyethylene, $\mathrm{CH}_{2}$, and $\mathrm{C}$ targets. Because of the limitations of the Bevalac injector, the highest energies are achievable only for the lightest projectiles. In addition, we were able to study the interactions of our lightest two projectile nuclei, krypton and xenon, only at their maximum Bevalac rigidity. Thus it must be noted that in any attempt to make a global fit, the mass and energy dependences are mixed and limited. The lightest nuclei were our only evenly charged heavy projectiles.

\section{ENERGY DEPENDENCE}

We can first consider whether our measurements are compatible with previous studies of fragmentation, ${ }^{7-9}$ which showed that the hypothesis of "limiting fragmentation" 10 provided a fair representation of the data at high $(>3-10 \mathrm{GeV})$ total kinetic energies of a light "projectile" nucleus as seen by a heavy "target" nucleus. These studies used light projectiles, protons or nuclei with $A \leq 40$, targets that covered the entire range of available mass, and looked, in general, at specific radioactive fragments with identified charge and mass. They showed that at sufficiently high total kinetic energies the cross sections tend to asymptotic values. In our studies, using heavy projectiles incident on relatively light targets, the relevant "available" energy is essentially the total kinetic energy of the target as seen in the rest frame of the projectile. ${ }^{11}$ In all cases, apart from the runs on targets that included hydrogen, and some of the lower-energy runs on a carbon target, these total energies should be sufficient to ensure that we were making measurements in the regime of limiting fragmentation. It is an unfortunate consequence of the limited energies per nucleon provided by the Bevalac that the data on hydrogen targets are not in the limiting fragmentation regime, unlike those on the heavier targets. It is consequently important to consider whether to attempt to organize the data in terms of the energy per nucleon of the projectile or in terms of the available energy for each projectile-target combination.

The effects of varying the available energy are shown in Figs. 1 and 2, which plot the cross sections as a function of $\Delta Z$ for $\mathrm{Au}$ and $\mathrm{La}$ projectiles incident on hydrogen and carbon targets at several different available energies. In both cases we see significant changes in the hydrogen cross sections as the energy, which is still significantly below the regime of limiting fragmentation, increases. On the other hand, the carbon cross sections are relatively independent of energy at energies where limiting fragmentation can be presumed to be applicable. The implication would be that the hydrogen cross sections tend to the carbon cross sections at high enough energies. Such a prediction is valuable given the unavailability of heavy projectiles at these high energies.

The Au plots for the hydrogen target in Fig. 1 are confused at larger $\Delta Z$ by the relatively large peak in the cross sections due to fission. The carbon cross sections show little evidence of the fission peak in such a plot. However this is more a consequence of the logarithmic nature of the cross section scale than evidence for a lack of fission. In Sec. IV D we will see that a linear plot re-

Au on $\mathrm{H}$ and $\mathrm{C}$

(at several total energies)

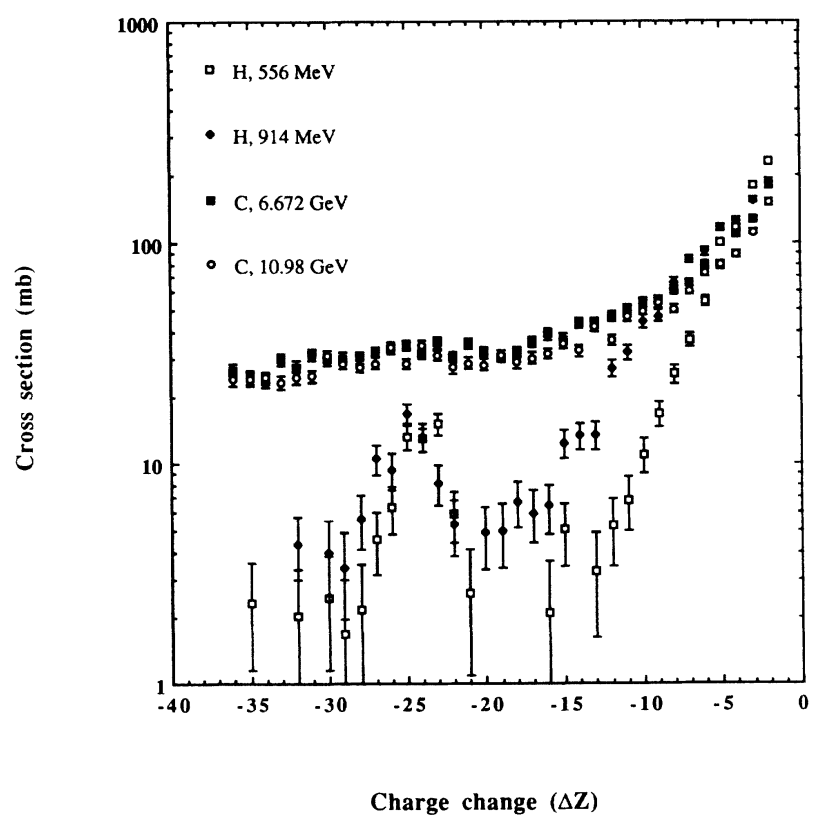

FIG. 1. Cross sections, in $\mathrm{mb}$, as a function of $\Delta Z$, for $\mathrm{Au}$ projectiles at several different total energies on hydrogen $\mathrm{H}$ and carbon $\mathrm{C}$ targets. 
La on $\mathrm{H}$ and $\mathrm{C}$

(at several total energies)

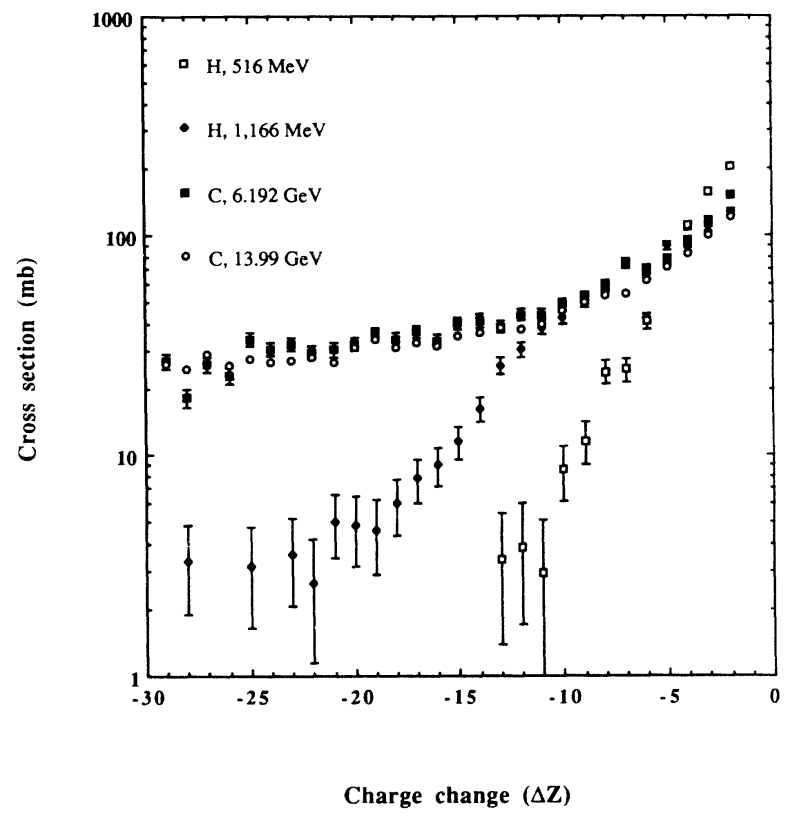

FIG. 2. Cross sections, in $\mathrm{mb}$, as a function of $\Delta Z$, for $\mathrm{La}$ projectiles at several different total energies on hydrogen and carbon targets.

veals a significant fission peak. Similarly, the apparently good agreement between the high- and low-energy carbon cross sections does not exclude a 20-30\% difference. Further support for the general validity of limiting fragmentation can be obtained by comparing low-energy projectiles on a light target such as carbon, with high-energy projectiles on a heavy target such as copper, thus covering the maximum range of available energies. Figures 3 and 4 plot the cross sections as a function of $\Delta Z$ for $\mathrm{Au}$ and $\mathrm{La}$ on carbon and copper. In both cases it can be seen that the cross sections are not strongly dependent on the target, the energy, or the projectile. Here again 20-30\% differences are not ruled out. There is also a small fission bump in the Au data at $|\Delta Z| \approx 25$ for both targets.

If limiting fragmentation is strictly applicable, then the cross sections for any defined group of fragments should be independent of energy and target, as long as the available energy exceeds some limiting value, one that from Figs. 1 and 2 appears to be $\leq 6 \mathrm{GeV}$. Since limiting fragmentation can be expected to be most applicable to the most extreme peripheral interactions, it can be tested by looking at fragments with small $\Delta Z$. Fragments with $\Delta Z=-1$ are a special case and have to be treated separately due to the Coulomb dissociations, Sec. IV A. Hence, we will define fragments with $|\Delta Z|$ between 2 and 6 as "small $\Delta Z$ " fragments and study the cross sections for Au projectile. Figure 5 shows these cross sections as a function of the available energy on targets of $\mathrm{C}, \mathrm{Al}$, and $\mathrm{Cu}$. Clearly there are significant dependencies on energy and target at the $20-30 \%$ level. If the same cross sections are plotted as a function of the energy per nucleon

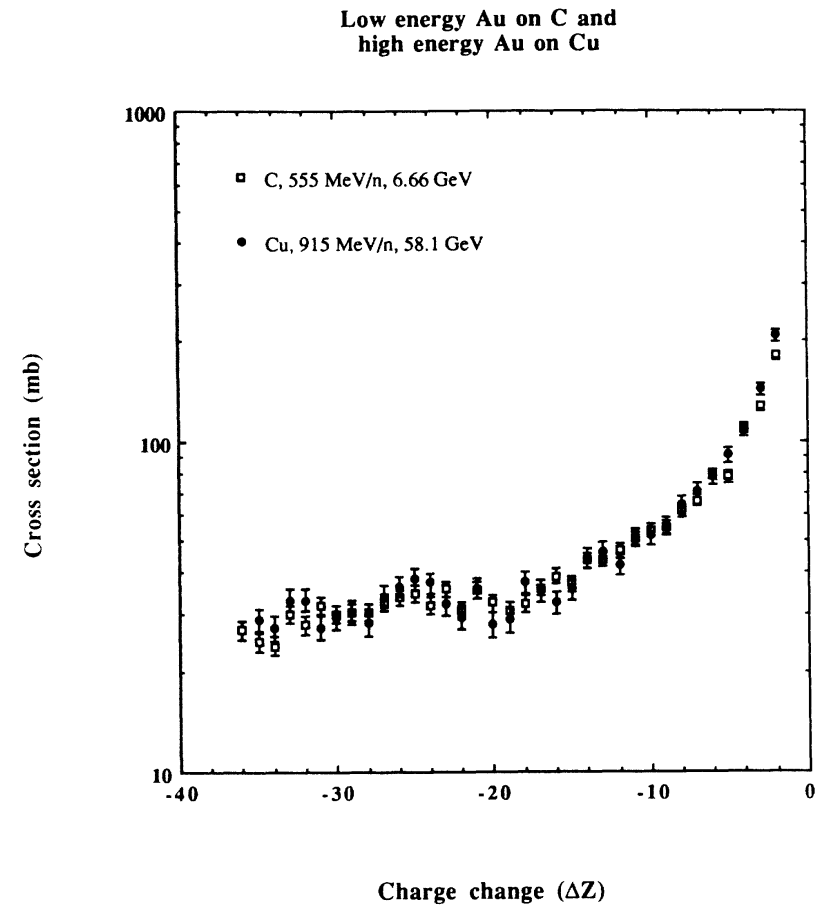

FIG. 3. Cross sections, in mb, as a function of $\Delta Z$, for low energy, $555 \mathrm{MeV} /$ nucleon, $\mathrm{Au}$ on $\mathrm{C}$ and high energy, 915 $\mathrm{MeV} /$ nucleon, $\mathrm{Au}$ and $\mathrm{Cu}$, covering a range of total energies from 6.66 to $58.1 \mathrm{GeV}$.

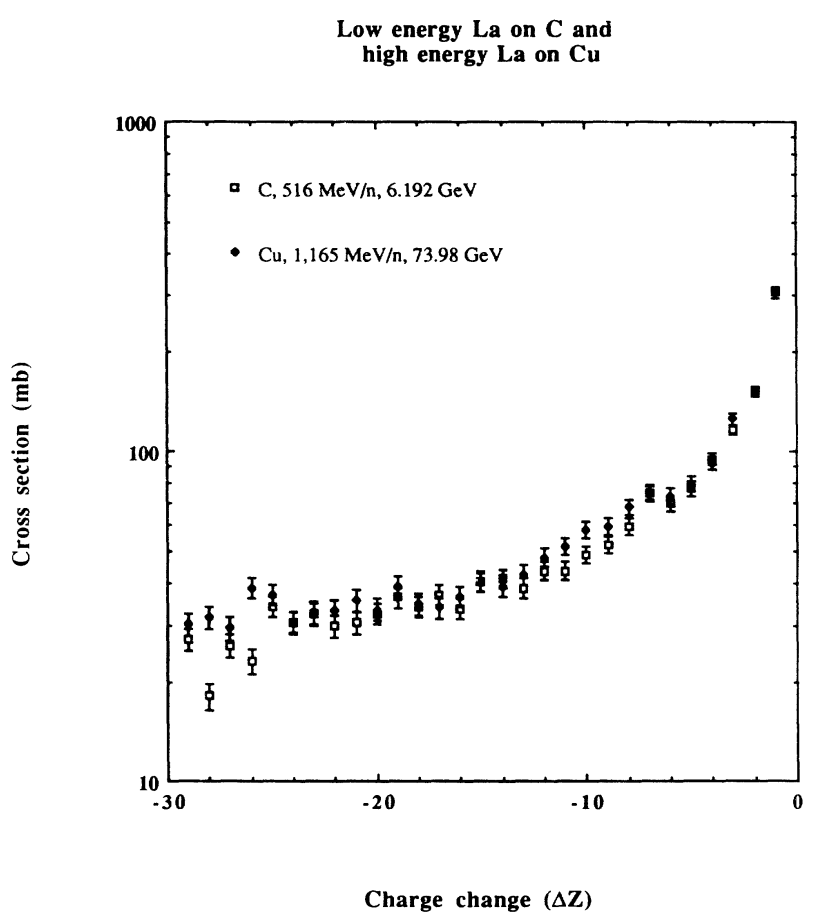

FIG. 4. Cross sections, in $\mathrm{mb}$, as a function of $\Delta Z$, for low energy, $516 \mathrm{MeV} /$ nucleon, La on $\mathrm{C}$ and high energy, 1165 $\mathrm{MeV} /$ nucleon, $\mathrm{La}$ on $\mathrm{Cu}$, covering a range of total energies from 6.192 to $73.98 \mathrm{GeV}$. 
Small $\Delta Z$ vs total target energies

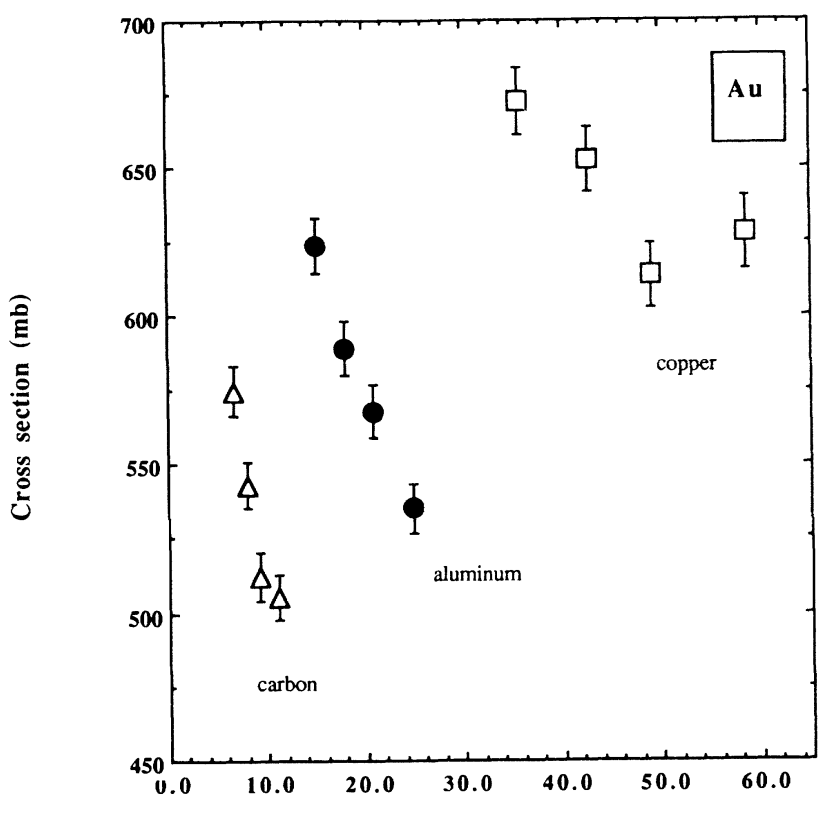

Total target energy (GeV)

FIG. 5. Cross sections summed over $2 \leq|\Delta Z| \leq 6$, in mb, as a function of total energy for a $A u$ projectile and small $\Delta Z$ fragments on targets of $\mathrm{C}, \mathrm{Al}$, and $\mathrm{Cu}$.

$E_{n}$ of the projectile, as in Fig. 6, the organization becomes more apparent, with cross sections that, at a given $E_{n}$ increase with target mass, and, for a particular target, decrease with increasing $E_{n}$. La projectiles show a similar pattern, Fig. 7. These target-dependent cross section variations are not simply due to the changing total cross sections. Figure 8 shows the fractional cross sections for the $\mathrm{Au}$ projectiles, obtained by dividing the small $\Delta Z$ cross sections by the total interaction cross sections, and shows that these fractions are greatest for the lighter targets. Thus lighter targets are more likely to cause fragments from a projectile with small charge changes than are heavier targets, a result that seems intuitively reasonable. In Fig. 9 we have compared these cross sections for $\mathrm{Au}$ and $\mathrm{La}$ projectiles on $\mathrm{C}$ and $\mathrm{Cu}$ targets as a function of the energy per nucleon of the projectiles. Although all these data are for available energies of greater than 6 $\mathrm{GeV}$, there are clear charge and energy dependencies. Hence, limiting fragmentation is an inadequate representation for data of this quality.

In what follows we will show that by expressing the data for the heavy targets in terms of the projectile energy per nucleon we can derive parametric fits that describe the charge and energy dependence to better than 10\% accuracy. We can also obtain parametric fits for the data on a hydrogen target which are nearly as accurate and are appreciably better than those provided by the available semiempirical fits. ${ }^{12}$ These parametric fits are also better than any we can obtain when the data are ex-
Small charge change cross sections (for $\Delta Z=2-6$ )

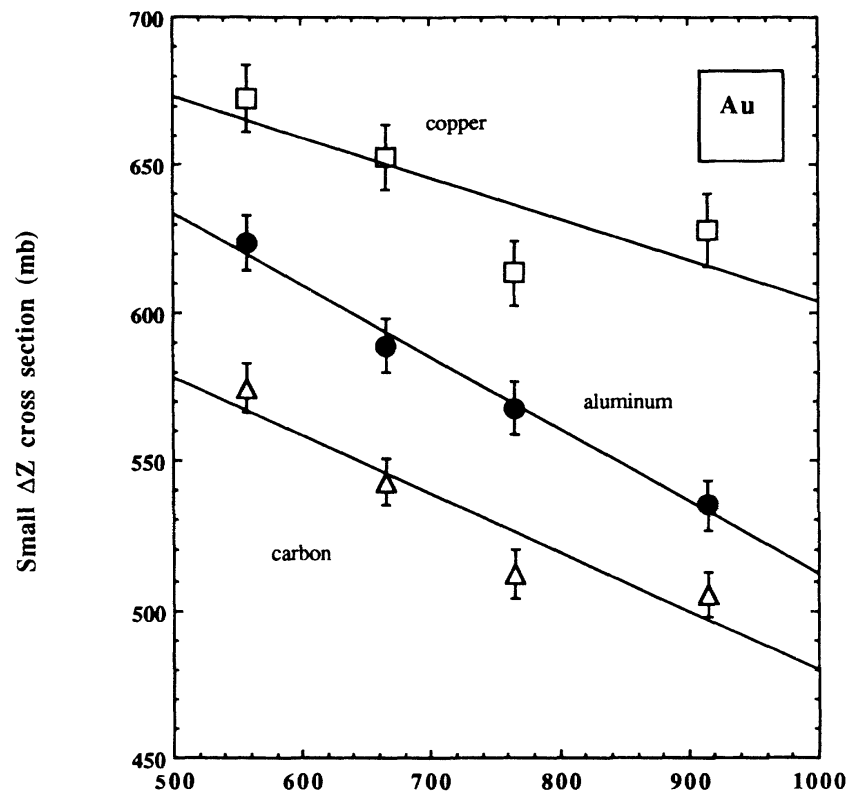

Projectile energy $(\mathrm{MeV} / \mathrm{n})$

FIG. 6. The data of Fig. 5 plotted as a function of energy per nucleon. Linear fits for each target are shown to guide the eye.

Small charge change cross sections (for $\Delta Z=2$ - 6)

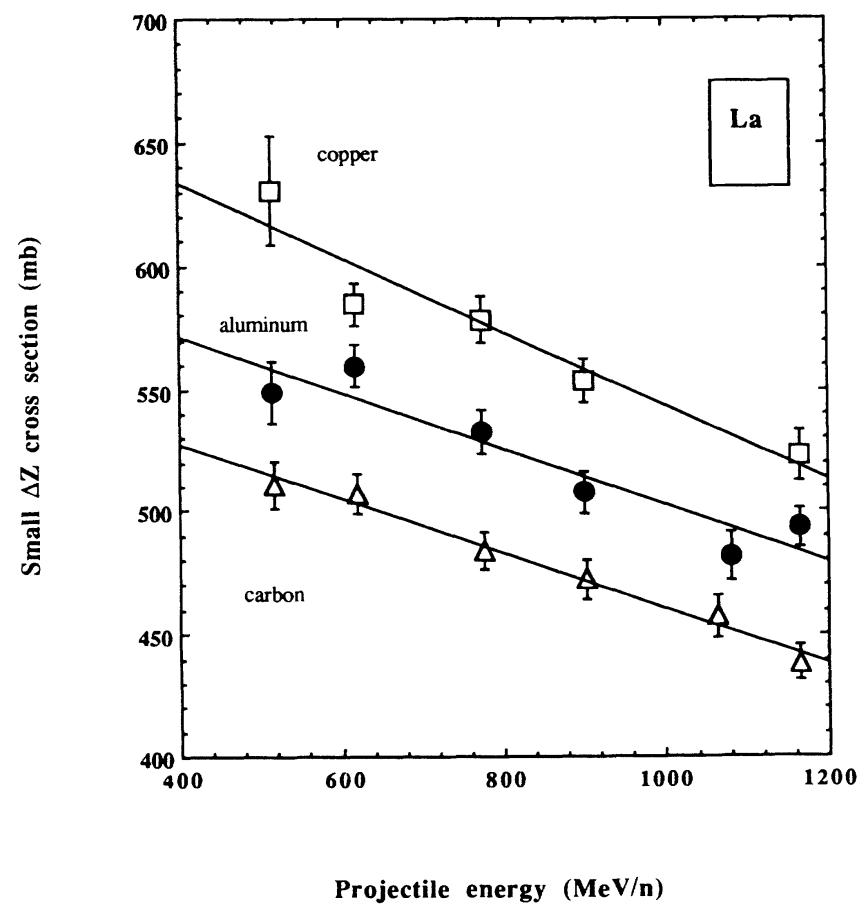

FIG. 7. Similar results to Fig. 6 for a La projectile. 
Fraction of total cross section for small charge change

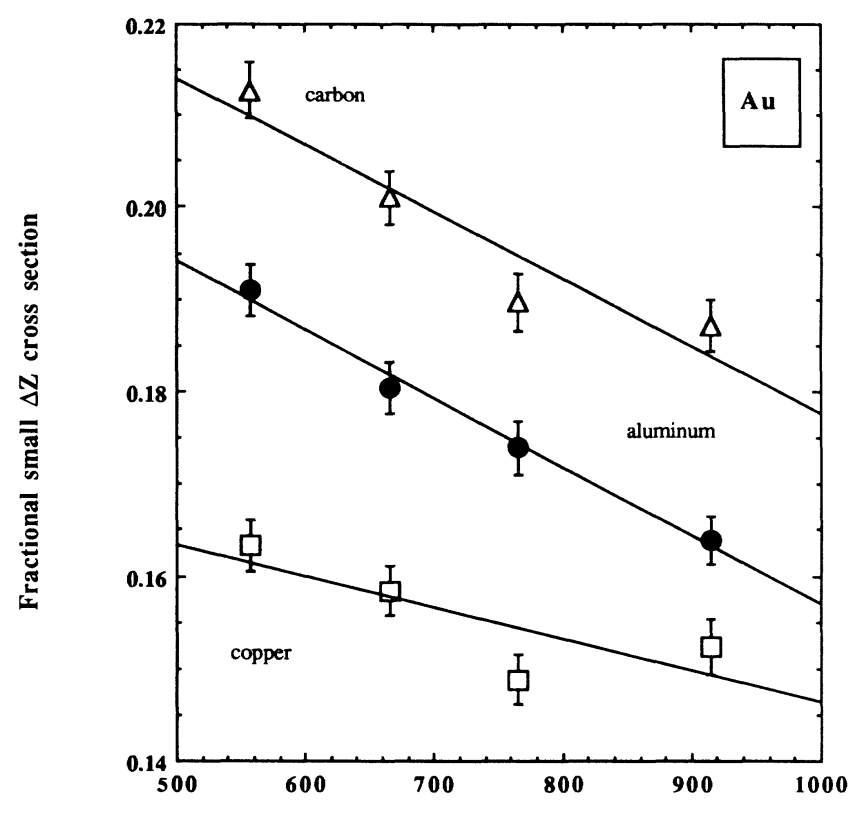

Projectile energy (MeV/n)

FIG. 8. The data of Fig. 5, with the cross sections normalized by the total charge-changing interaction cross sections.

$\mathrm{Au}$ and $\mathrm{La}$ on $\mathrm{Cu}$ and $\mathrm{C}$ targets

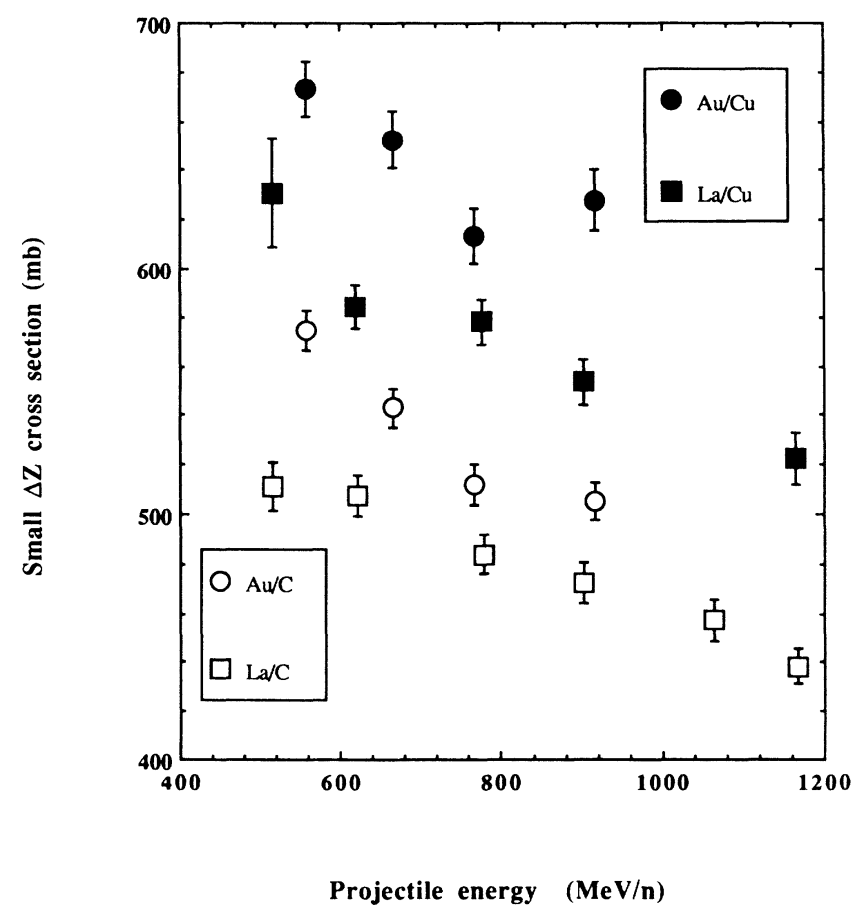

FIG. 9. Cross sections summed over $2 \leq|\Delta Z| \leq 6$, in mb, as a function of energy per nucleon, for $\mathrm{Au}$ and $\mathrm{La}$ on $\mathrm{Cu}$ and $\mathrm{C}$ targets. pressed in terms of the total energy. However, the hypothesis of limiting fragmentation can still provide a useful indication of the expected cross sections for hydrogen targets at higher energies until better experimental values become available.

\section{A GLOBAL FIT TO THE CROSS SECTIONS}

\section{A. Introduction}

Ideally we can hope to find global equations that will provide an acceptable fit to all the measured cross sections, but do not depend on an excessively large number of arbitrary parameters. To be useful these equations should provide better fits to the data and be simpler than the semiempirical equations developed previously by Silberberg et al. ${ }^{12}$ In searching for these equations, our earlier experiment ${ }^{5}$ yielded two important results which we have developed further in this analysis. First, for heavyprojectile nuclei interacting with heavy targets, the fragments with relatively small negative charge changes from the incident nuclei have cross sections which are excellently described by power laws in $|\Delta Z|$. These power-law dependencies should be clearly distinguished from the steep power-law dependencies that are seen in the multifragmentation region for fragments with $A \leq 50,{ }^{13,14}$ which is a region not studied in this experiment. Second, heavy projectiles on hydrogen targets have cross sections which show a somewhat less regular exponential dependence on $|\Delta Z|$. These relationships are described by Eqs. (1) and (2), respectively, which, like all the following equations, give the cross sections in $\mathrm{mb}$ :

$$
\begin{aligned}
& \sigma_{t}(\Delta Z)=\sigma_{\beta}|\Delta Z|^{-\beta}, \\
& \sigma_{H}(\Delta Z)=\sigma_{\delta} \exp -(|\Delta Z| / \delta),
\end{aligned}
$$

where the fitting parameters $\sigma_{\beta}, \beta, \sigma_{\delta}$, and $\delta$ are functions of energy and the masses of the projectile and target nuclei.

There are several possible ways to attempt to characterize these dependences. We can follow the approach of several earlier groups, who have used modifications of the original six-parameter equations proposed by Rudstam. ${ }^{15}$ Cumming et al. ${ }^{16}$ fitted their data for ${ }^{14} \mathrm{~N}$ and protons on $\mathrm{Cu}$ in such a way, while Porile et al. ${ }^{9}$ used ten-parameter fits to represent the cross sections of products with $A=20-100$ from the interaction of ${ }^{12} \mathrm{C}$ ions and protons with a silver target. This approach leads eventually to the multiparametric semiempirical relations of Silberberg et al., ${ }^{11}$ which we have found previously to give predictions as much as a factor of two in error. Consequently we have attempted a new approach, which does not assume limiting fragmentation, but does use the concept of factorization. ${ }^{16}$ In particular, it has been shown by Olson et al. ${ }^{17}$ that for projectiles of iron and lighter nuclei the projectile and target dependences can be separated by "factorization," and it seems reasonable to attempt to apply the same principle to our heavier projectiles. Our objective was to attempt to find a universal parametric fit with a small number of independent parameters that would be applicable over a wide range of projectiles, targets, and energies. 
In the analysis discussed here we will refer to fitting parameters, defined as the parameters adjusted by a fitting program as it seeks the best fit to a data set. In the equations used, these are written as $p_{i}$ and $q_{i}$, depending on the equation, with $p$ for heavy projectile and target, $q$ for heavy projectile and hydrogen target. The projectile energy, mass and charge, the target mass and charge, and $\Delta Z$ were all treated as input parameters. We used two criteria for determining goodness of fit. Primarily, we used the reduced chi squared value $\chi^{2}$. Secondarily, if a fitting parameter in an equation had a large uncertainty and a large covariance with other parameters, indicating that it had little effect on the goodness of the fit, we modified the equations to remove the dependence on that parameter and the corresponding input variable. This does not necessarily mean that there was no dependence, but that the dependence for the cross sections, in our limited data set, was too weak for the form of the dependence to be defined. We generally chose the simplest equation that followed the form of the dependence of the cross sections on the input parameters and had a $\chi^{2}$ value insignificantly different from the best value. The fitting program used was based on the algorithms described by Bevington, ${ }^{18}$ with the uncertainties quoted being derived from the diagonal elements of the inverse of the curvature matrix. These fitting procedures, and the various relationships studied, are discussed in greater detail in the thesis by Cummings. ${ }^{24}$

\section{B. Factorization}

Factorization is based on the concept that the cross section for a given projectile and target can be separated into a factor that is independent of the target and a factor that is independent of the fragment. ${ }^{16,19,20}$ Then the cross section for the production of a fragment $F$ from a projectile nucleus $P$ interacting with a target nucleus $T$ can be written

$$
\sigma(F, P, T)=\gamma(F, P) \eta(P, T) .
$$

Here $\gamma(F, P)$ is a factor which depends only on the species of projectile and fragment but not the target, and $\eta(P, T)$ is a factor which depends only on the species of projectile and target but not on the fragment. Equations which follow this format are said to follow "weak factorization" as opposed to "strong factorization" which restricts the dependence of the second factor to the target only. Equation (3) is taken from Olson et al., ${ }^{17}$ where three theoretical models or theories were compared to this equation. These were an opaque disk geometrical model of colliding nuclei, abrasion-ablation theory, and an excitation-decay model. That paper reported on the factorization of cross sections for fragmentation of projectiles of carbon $(Z=6)$ up to iron $(Z=26)$ incident on nuclear targets of various masses, not including hydrogen.

In an opaque-disk model, $\eta(P, T)$ is proportional to an impact term of the form $\left(A_{P}^{1 / 3}+A_{T}^{1 / 3}\right)$, the combined radii of the projectile nucleus and target nucleus, and $\gamma(F, P)$ is the width of an annular band on a disk of this radius and contains all the details of the physics, but no dependence on the target. In the abrasion-ablation theory, when two high-energy nuclei collide, the overlapping portion of their volumes is sheared away (abrasion) and the remaining spectator portion of the projectile nucleus is excited, causing the further loss of some nucleons through decay (ablation). The cross section equation for abrasion-ablation violates factorization because the fragmentation channel is dependent on the impact parameter and so on the target. ${ }^{21,22}$ The excitation-decay model is developed by Olson et al. ${ }^{17}$ from a concept suggested by Karol. $^{23}$ It follows factorization, with $\eta(P, T)$ proportional to the sum of the radii of the target and projectile nuclei plus a constant, and $\gamma(F, P)$ proportional to an integral over excitation energies of the probability for producing a given energy in a collision involving one nucleon in the projectile nucleus, times the probability for that level of excitation to produce the given fragment. If our data on these much heavier projectile nuclei were consistent with factorization, this would be an argument against the abrasion-ablation model.

\section{Fits to the heavy target data}

The first step in obtaining a global representation was to fit the cross sections for charge-changing interactions over a limited range of values of $\Delta Z$ for each projectiletarget-energy combination with the form of Eq. (1). The limited range $2 \leq|\Delta Z| \leq 20$ was chosen for three practical reasons. First, experimental cross sections were measured over this range for all the runs, apart from the two runs of krypton on aluminum (only to $\Delta Z=-18$ ) and low-energy holmium on aluminum (only to $\Delta Z=-19$ ). Second, for $\mathrm{Au}$ the signals from fragments with $|\Delta Z| \leq 20$ are not yet confused with the signals from fission fragments. Finally, we have observed that the $\Delta Z=-1$ cross sections are systematically larger than expected from the trend of the cross sections for larger charge changes. Hence we conclude that the processes leading to $\Delta Z=-1$ fragments appear to include an additional component, probably attributable to electromagnetic dissociation, yielding a larger than expected cross section, Sec. IV A. Table I contains the results of the least-squares fit of Eq. (1) for each run. Observe that $\chi^{2}$ for these runs is, in almost all cases, close to unity, indicating that the measured cross sections are consistent with the power law to within the quoted uncertainties.

The next step in the determination of a global fit was to study the projectile, target, and energy dependences of the power-law parameters $\sigma_{\beta}$ and $\beta$. It was apparent from a study of the variations of these parameters with the different variables ${ }^{24}$ that $\sigma_{\beta}$ has an obvious, but poorly defined, dependence on all three variables and that $\beta$ depends mainly on energy and only weakly on projectile and target over the range studied.

If factorization is applicable, we should be able to separate the equations for the cross sections into factors, one dependent on the projectile and fragment alone, and one dependent on the projectile and on an impact term of the form $\left(A_{P}^{1 / 3}+A_{T}^{1 / 3}-\epsilon\right)$, where $\epsilon$ represents an overlap. Then $\sigma_{\beta}$ should contain all the target dependence. The power of $|\Delta Z|, \beta$, would then be the projectile- 
fragment-dependent factor. In factorization, either factor may have an energy dependence. If the interactions are truly peripheral in nature, the cross sections should be proportional to the impact term, while if the interactions involve many nucleons, the cross sections should be proportional to an area or to the square of the impact term.

To find the global fit we chose general equations incorporating these principles with nine variable parameters $p_{i}=p_{1}$ to $p_{9}$. Details of these general equations and the different variations that have been tried, with the goodness of fits obtained, are given elsewhere. ${ }^{24}$ We assumed that $\sigma_{\beta}$ has the form expected for peripheral interactions, and either a linear or power-law dependence on the masses and energy of the projectile nuclei. Similarly $\beta$ can have either linear or power-law dependences. These equations also allowed for a variation of the exponent of the impact term. If the cross sections are factorizable, the target dependence of the exponent $\beta$ should vanish, but it does not. The principle of factorization was formulated, however, using mostly data from fragmentation of lighter projectiles and need not, a priori, apply here. These general equations could be readily fitted to the data with reduced $\chi^{2}$ of appreciably less than 2.0. We did also try to use the hypothesis of limiting fragmentation to make fits based on the total energy, but were unable to find any with values of $\chi^{2}$ less than 2.5 , showing that better fits can be obtained by not using this hypothesis.

TABLE I. Power-law fits to the heavy target data for all the projectiles, targets, and energies used in this study.

\begin{tabular}{|c|c|c|c|c|c|c|c|}
\hline & $\begin{array}{c}\text { Beam } \\
\text { mass }\end{array}$ & & $\begin{array}{c}\text { Target } \\
\text { mass }\end{array}$ & $\begin{array}{c}\text { Energy } \\
(\mathrm{MeV} / \text { nucleon })\end{array}$ & $\sigma(\mathrm{mb})$ & $\beta$ & Reduced $\chi^{2}$ \\
\hline $\mathrm{Kr}$ & 84 & $\mathrm{Al}$ & 27.0 & 1418 & $162.4 \pm 8.2$ & $0.53 \pm 0.02$ & 0.70 \\
\hline $\mathrm{Xe}$ & 131 & $\mathrm{C}$ & 12.0 & 1182 & $200.5 \pm 8.3$ & $0.66 \pm 0.02$ & 1.02 \\
\hline $\mathrm{Xe}$ & 131 & $\mathrm{Al}$ & 27.0 & 1155 & $185.4 \pm 11.8$ & $0.58 \pm 0.03$ & 0.76 \\
\hline $\mathbf{L a}$ & 139 & $\mathrm{C}$ & 12.0 & 516 & $245.7 \pm 8.8$ & $0.69 \pm 0.02$ & 1.18 \\
\hline $\mathbf{L a}$ & 139 & $\mathrm{C}$ & 12.0 & 621 & $242.4 \pm 7.5$ & $0.69 \pm 0.02$ & 0.80 \\
\hline $\mathrm{La}$ & 139 & $\mathrm{C}$ & 12.0 & 777 & $228.6 \pm 6.9$ & $0.67 \pm 0.01$ & 1.61 \\
\hline $\mathrm{La}$ & 139 & $\mathrm{C}$ & 12.0 & 903 & $213.5 \pm 7.0$ & $0.65 \pm 0.02$ & 1.46 \\
\hline $\mathrm{La}$ & 139 & $\mathrm{C}$ & 12.0 & 1065 & $216.7 \pm 7.7$ & $0.69 \pm 0.02$ & 1.42 \\
\hline $\mathrm{La}$ & 139 & $\mathrm{C}$ & 12.0 & 1166 & $193.7 \pm 6.0$ & $0.63 \pm 0.02$ & 0.80 \\
\hline $\mathrm{La}$ & 139 & Al & 27.0 & 515 & $265.0 \pm 11.6$ & $0.70 \pm 0.02$ & 2.05 \\
\hline $\mathbf{L a}$ & 139 & $\mathrm{Al}$ & 27.0 & 618 & $275.9 \pm 8.0$ & $0.71 \pm 0.01$ & 1.51 \\
\hline $\mathrm{La}$ & 139 & $\mathrm{Al}$ & 27.0 & 775 & $257.3 \pm 8.1$ & $0.70 \pm 0.02$ & 1.26 \\
\hline $\mathrm{La}$ & 139 & Al & 27.0 & 901 & $238.4 \pm 7.7$ & $0.67 \pm 0.02$ & 1.31 \\
\hline $\mathrm{La}$ & 139 & $\mathrm{Al}$ & 27.0 & 1080 & $236.6 \pm 9.1$ & $0.70 \pm 0.02$ & 2.51 \\
\hline $\mathrm{La}$ & 139 & $\mathrm{Al}$ & 27.0 & 1165 & $227.1 \pm 6.9$ & $0.66 \pm 0.01$ & 1.61 \\
\hline $\mathrm{La}$ & 139 & $\mathrm{Cu}$ & 63.5 & 515 & $317.7 \pm 20.7$ & $0.72 \pm 0.03$ & 0.99 \\
\hline $\mathrm{La}$ & 139 & $\mathrm{Cu}$ & 63.5 & 618 & $279.4 \pm 8.1$ & $0.69 \pm 0.01$ & 1.21 \\
\hline $\mathrm{La}$ & 139 & $\mathrm{Cu}$ & 63.5 & 775 & $274.1 \pm 8.3$ & $0.68 \pm 0.01$ & 0.45 \\
\hline $\mathbf{L a}$ & 139 & $\mathrm{Cu}$ & 63.5 & 901 & $266.2 \pm 8.3$ & $0.69 \pm 0.02$ & 2.00 \\
\hline $\mathbf{L a}$ & 139 & $\mathrm{Cu}$ & 63.5 & 1165 & $246.5 \pm 9.4$ & $0.67 \pm 0.02$ & 1.41 \\
\hline Ho & 165 & $\mathrm{C}$ & 12.0 & 489 & $268.8 \pm 7.1$ & $0.70 \pm 0.01$ & 2.13 \\
\hline Ho & 165 & $\mathrm{C}$ & 12.0 & 772 & $250.8 \pm 6.9$ & $0.66 \pm 0.01$ & 0.32 \\
\hline Ho & 165 & $\mathrm{C}$ & 12.0 & 922 & $225.8 \pm 7.4$ & $0.66 \pm 0.02$ & 1.80 \\
\hline Ho & 165 & $\mathrm{C}$ & 12.0 & 1048 & $228.6 \pm 9.9$ & $0.70 \pm 0.02$ & 0.63 \\
\hline Ho & 165 & Al & 27.0 & 489 & $321.4 \pm 10.5$ & $0.76 \pm 0.02$ & 2.29 \\
\hline Ho & 165 & Al & 27.0 & 772 & $270.0 \pm 7.7$ & $0.67 \pm 0.01$ & 1.60 \\
\hline Ho & 165 & Al & 27.0 & 1024 & $227.4 \pm 12.7$ & $0.66 \pm 0.03$ & 0.60 \\
\hline Ho & 165 & $\mathrm{Cu}$ & 63.5 & 772 & $304.3 \pm 9.2$ & $0.69 \pm 0.01$ & 1.26 \\
\hline $\mathrm{Au}$ & 197 & $\mathrm{C}$ & 12.0 & 557 & $298.1 \pm 8.4$ & $0.76 \pm 0.01$ & 1.32 \\
\hline $\mathrm{Au}$ & 197 & $\mathrm{C}$ & 12.0 & 666 & $274.4 \pm 7.7$ & $0.74 \pm 0.01$ & 1.22 \\
\hline $\mathrm{Au}$ & 197 & $\mathrm{C}$ & 12.0 & 766 & $241.1 \pm 7.4$ & $0.68 \pm 0.02$ & 0.89 \\
\hline $\mathrm{Au}$ & 197 & $\mathrm{C}$ & 12.0 & 915 & $255.0 \pm 7.3$ & $0.74 \pm 0.01$ & 1.69 \\
\hline $\mathrm{Au}$ & 197 & $\mathrm{C}$ & 12.0 & 961 & $221.2 \pm 10.2$ & $0.70 \pm 0.02$ & 1.01 \\
\hline $\mathrm{Au}$ & 197 & $\mathrm{Al}$ & 27.0 & 557 & $330.3 \pm 9.3$ & $0.77 \pm 0.01$ & 0.63 \\
\hline $\mathrm{Au}$ & 197 & Al & 27.0 & 666 & $317.2 \pm 9.4$ & $0.79 \pm 0.02$ & 1.48 \\
\hline $\mathrm{Au}$ & 197 & $\mathrm{Al}$ & 27.0 & 766 & $284.8 \pm 8.9$ & $0.73 \pm 0.02$ & 1.41 \\
\hline $\mathrm{Au}$ & 197 & Al & 27.0 & 915 & $269.6 \pm 8.1$ & $0.73 \pm 0.02$ & 1.37 \\
\hline $\mathrm{Au}$ & 197 & $\mathrm{Al}$ & 27.0 & 933 & $246.9 \pm 14.4$ & $0.72 \pm 0.03$ & 0.62 \\
\hline $\mathrm{Au}$ & 197 & $\mathrm{Cu}$ & 63.5 & 559 & $358.4 \pm 11.6$ & $0.78 \pm 0.02$ & 1.69 \\
\hline $\mathrm{Au}$ & 197 & $\mathrm{Cu}$ & 63.5 & 667 & $366.9 \pm 12.1$ & $0.82 \pm 0.02$ & 1.32 \\
\hline $\mathrm{Au}$ & 197 & $\mathrm{Cu}$ & 63.5 & 767 & $304.5 \pm 10.7$ & $0.73 \pm 0.02$ & 0.88 \\
\hline $\mathrm{Au}$ & 197 & $\mathrm{Cu}$ & 63.5 & 915 & $357.7 \pm 13.6$ & $0.83 \pm 0.02$ & 0.89 \\
\hline
\end{tabular}


Fits that are almost equally good as those from the general nine-parameter equations can be obtained by assuming that while $\sigma_{\beta}$ has power-law dependences on mass and energy, $\beta$ has linear dependences. However, the resulting best-fit values of the parameters give equations that appear less physical. For example, the lowest$\chi^{2}$ values are obtained if the impact term is assumed to have a negative overlap. We were unable to obtain fits that converged if we assumed that $\beta$ had a power-law dependence on mass and energy.

We examined the results of using various modifications of these equations, and from these selected as "preferred" the one which appeared to be the most physical and had the fewest free parameters, but still gave an acceptable value of $\chi^{2}$. It was found that retaining a weak dependence of $\beta$ on target mass improved the fit without introducing large uncertainties in the values of the parameters. Including a dependence of $\sigma_{B}$ on projectile mass other than that in the impact term did not appreciably improve the fit. The resulting equation has only seven free parameters:

$$
\begin{aligned}
\sigma\left(A_{P},\right. & \left.A_{T}, E, \Delta Z\right) \\
= & p_{1}\left(A_{P}^{1 / 3}+A_{T}^{1 / 3}-p_{2}\right)\left(1+\frac{E}{p_{4}}\right) \\
& \times|\Delta Z|^{\left\{p_{6}\left[1+\left(A_{P} / p_{7}\right)\right]\left[1+\left(A_{T} / p_{8}\right)\right]\left[1+\left(E / p_{9}\right)\right]\right\}} .
\end{aligned}
$$

Minimizing $\chi^{2}$ gives values for these parameters of $p_{1}=45.24 \pm 2.15 ; \quad p_{2}=0.81 \pm 0.36 ; \quad p_{4}=-3.48 \pm 0.16$; $p_{6}=-0.614 \pm 0.013 ; \quad p_{7}=788.7 \pm 59.5 ; \quad p_{8}=1173 \pm 204 ;$ and $p_{9}=-11.13 \pm 1.72$. This equation has a $\chi^{2}$ of 1.81 , insignificantly greater than those for the more general nine-parameter equations and appears somewhat more physical. $^{24}$

Equation (4) is able to fit every single one of the 795 cross sections in our $2 \leq|\Delta Z| \leq 20$ data set to within a factor of 1.28. Furthermore, the values derived for the parameters have considerably smaller formal errors than in the nine-parameter fit. Figure 10 shows an example of the fit to the data from one run, $\mathrm{Au}$ on $\mathrm{C}$ at 766 $\mathrm{MeV} /$ nucleon. Figure 11 shows the distribution of the normalized residuals between the values calculated from Eq. (4) and the data points. This distribution is well characterized by a Gaussian peak with a standard deviation of 0.072 . There are minor tails on either side of the peak, but $95 \%$ of all the values lie within \pm 0.14 . We were unable to identify any obvious regular residual dependences on any of the input parameters.

It is unlikely that our present best overall fits can be improved without additional data, particularly data which better define the variation of cross sections with type of projectile nucleus. The largest deviations from the overall fit are for the projectiles with extremes in mass. This equation yields values too small for the cross sections of krypton, which also has the highest energy in the data set and is an even charged projectile nucleus. The cross sections of gold are over- or underestimated in a manner which depends on energy. Studies over a wider range of target masses would help to clarify the validity of factorization for these heavy nuclei. Nevertheless,

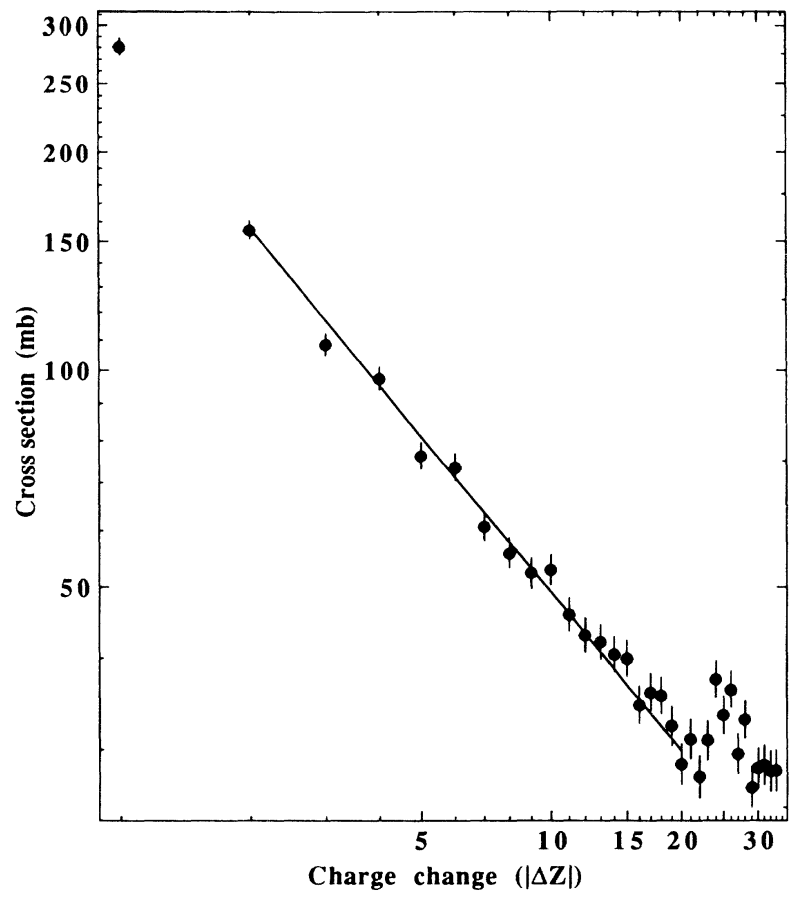

FIG. 10. Fit of Eq. (4) to the measured cross sections of $\mathrm{Au}$ on $\mathrm{C}$ at $766 \mathrm{MeV} /$ nucleon, plotted as a function of charge change $\Delta Z$. The fission peak can be clearly seen.

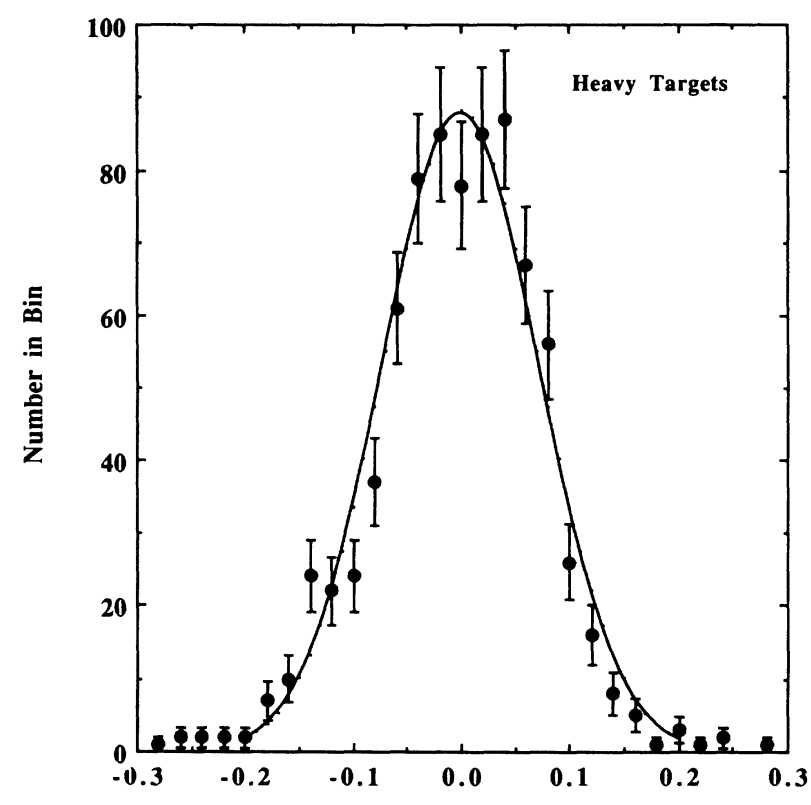

(Fit-Measured)/Fit

FIG. 11. Normalized residuals, formed from the difference between the calculated and measured values of cross sections from Eq. (4), divided by the calculated values. A Gaussian function of equal area to the data, with a standard deviation of 0.072 , is also shown. 
overall Eq. (4) provides a remarkably good fit over its quoted range of validity, and is marginally the best of those we have examined.

\section{Fits to the hydrogen target data}

In a manner similar to the heavy target analysis, the first step in finding a global fit to the hydrogen target data is determining the basic fitting equation for each run, which in this case is Eq. (2), an exponential in $\Delta Z$. The results of the least-square fit to the cross sections of $2 \leq|\Delta Z| \leq 20$ interactions for the 16 available runs are found in Table II. Reduced $\chi^{2}$ s for these runs varied widely, with an average of about 3 . Deviations of the data from the fit to Eq. (2) indicate that for the small $|\Delta Z|(\leq 8)$ there may be an odd-even effect in the cross sections of the order of a few percent. Here, "odd-even effect" means that the production of the even-charged fragments is enhanced. Most of the runs show a slight break in the slope of the exponential at around $\Delta Z=-7$ to -8 . Fitting the data to a modified form of Eq. (2) with a double-slope exponential yields much better (smaller) values of $\chi^{2}$ :

$$
\begin{array}{r}
\sigma=\sigma_{\delta} \exp [-(|\Delta Z| / \delta)] \text { for }|\Delta Z| \leq\left|\Delta Z_{\mathrm{C}}\right| \\
\sigma=\sigma_{\delta} \exp \left[-\left(\left|\Delta Z_{\mathrm{C}}\right| / \delta\right)\right] \exp \left\{-\left[\left(|\Delta Z|-\left|\Delta Z_{\mathrm{C}}\right|\right) / \delta^{\prime}\right]\right\} \\
\text { for }|\Delta Z|>\left|\Delta Z_{\mathrm{C}}\right|
\end{array}
$$

The parameters and $\chi^{2}$, $s$ for the fit of this equation to each individual run with a fixed $\Delta Z_{C}$ of 7.5 are listed in Table III. The individual $\chi^{2}$,s are on the average about 1.5. The dependence of the parameters $\sigma_{\delta}, \delta$, and $\delta^{\prime}$ on projectile mass and energy are illustrated in Figs. 12, 13, and 14. Clearly there is a well-defined power-law dependence of $\delta$ and $\delta^{\prime}$ on energy, and no significant dependence on projectile mass. Although the dependence of the parameters on projectile mass and energy looks regu-

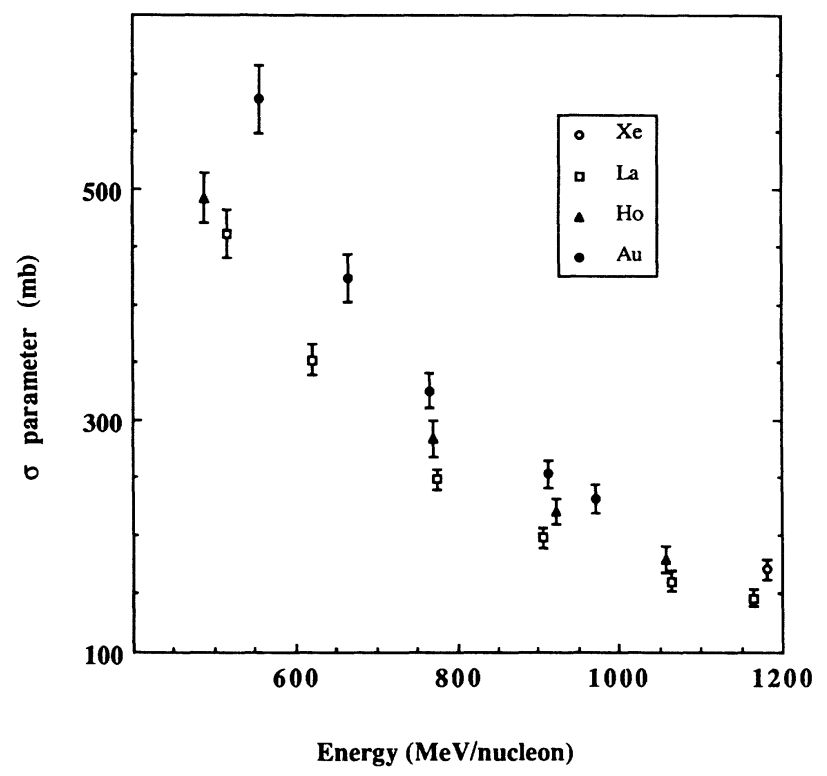

FIG. 12. The energy and projectile dependence of the $\sigma_{\delta}$ parameter in Eq. (5).

lar, the reduced $\chi^{2}$,s for these fits are significantly greater than one, so the exponential fits are not a perfect representation of the data. The dependences of $\sigma_{\delta}$ on projectile mass and energy and of $\delta$ and $\delta^{\prime}$ on energy defined our initial choice for a general global fit equation which takes into account a possible odd-even effect. This equation has nine variable parameters. Fourteen combinations and permutations of the basic equation were tried without producing any improved fits, but in some cases a reduced number of free parameters produced fits almost equally good. ${ }^{24}$ There appears to be a lower limit to the $\chi^{2}$ value we can achieve with our data and the assumption of a double-sloped exponential fit. One could introduce many terms to cover special cases, but these would not be likely to apply to other unmeasured combinations

TABLE II. Exponential fits to the hydrogen target data for all the projectiles and energies used in this study.

\begin{tabular}{lccccc}
\hline \hline & $\begin{array}{c}\text { Beam } \\
\text { mass }\end{array}$ & $\begin{array}{c}\text { Energy } \\
(\mathrm{MeV} / \text { nucleon })\end{array}$ & $\sigma(\mathrm{mb})$ & $\delta$ & Reduced $\chi^{2}$ \\
\hline $\mathrm{Xe}$ & 131 & 1182 & $191.3 \pm 6.0$ & $6.32 \pm 0.16$ & 2.12 \\
$\mathrm{La}$ & 139 & 516 & $477.6 \pm 18.9$ & $2.56 \pm 0.07$ & 1.97 \\
$\mathrm{La}$ & 139 & 621 & $379.7 \pm 12.2$ & $3.09 \pm 0.07$ & 1.10 \\
$\mathrm{La}$ & 139 & 776 & $304.7 \pm 8.2$ & $3.96 \pm 0.08$ & 7.10 \\
$\mathrm{La}$ & 139 & 906 & $256.6 \pm 7.3$ & $4.61 \pm 0.10$ & 7.62 \\
$\mathrm{La}$ & 139 & 1065 & $191.4 \pm 6.8$ & $5.91 \pm 0.17$ & 2.48 \\
$\mathrm{La}$ & 139 & 1166 & $186.2 \pm 5.5$ & $6.17 \pm 0.15$ & 5.41 \\
$\mathrm{Ho}$ & 165 & 488 & $509.3 \pm 19.3$ & $2.54 \pm 0.06$ & 1.62 \\
$\mathrm{Ho}$ & 165 & 772 & $348.7 \pm 12.8$ & $4.05 \pm 0.09$ & 3.04 \\
$\mathrm{Ho}$ & 165 & 922 & $296.4 \pm 9.3$ & $4.86 \pm 0.10$ & 4.92 \\
$\mathrm{Ho}$ & 165 & 1057 & $219.1 \pm 7.9$ & $6.05 \pm 0.16$ & 1.84 \\
$\mathrm{Au}$ & 197 & 557 & $591.2 \pm 25.3$ & $2.56 \pm 0.06$ & 2.73 \\
$\mathrm{Au}$ & 197 & 665 & $490.3 \pm 18.1$ & $3.09 \pm 0.07$ & 2.72 \\
$\mathrm{Au}$ & 197 & 766 & $406.2 \pm 13.2$ & $3.80 \pm 0.08$ & 4.94 \\
$\mathrm{Au}$ & 197 & 914 & $315.1 \pm 9.3$ & $4.73 \pm 0.09$ & 3.92 \\
$\mathrm{Au}$ & 197 & 972 & $283.1 \pm 8.7$ & $5.10 \pm 0.10$ & 2.34 \\
\hline \hline
\end{tabular}




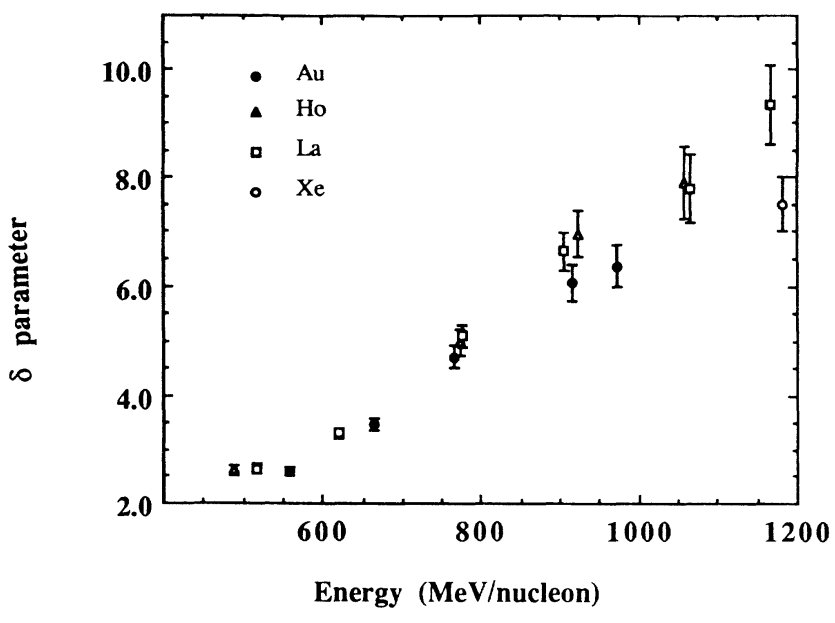

FIG. 13. The energy and projectile dependence of the $\delta$ parameter in Eq. (5).

of projectile, energy, and target. The deviations from the fits show little regularity, though the $\chi^{2}$ values of about 2 indicate that a double-sloped exponential is not quite the correct function.

The exact position of the break in the slope does not appear to affect the goodness of fit very much, nor it it well determined if allowed to vary in a fit of Eq. (5) to individual runs. However, it consistently converges to between 7.5 and 7.95 for any general expression of a double-sloped exponential fit to the whole data set. The larger charge changes have cross sections that are of diminishing size and astrophysical importance, and the fit of the first half of the exponential to the small charge changes is very good.

Removing the odd-even factor does not harm the fit significantly, nor does making $\delta^{\prime}$ and $\delta$ have the same energy dependence. The resulting equation has only seven free parameters:

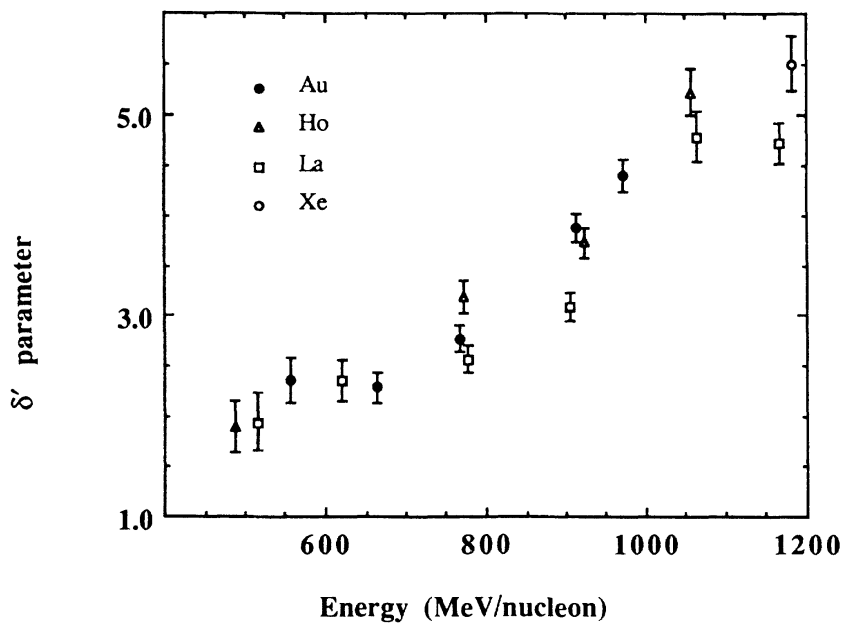

FIG. 14. The energy and projectile dependence of the $\delta^{\prime}$ parameter in Eq. (5).

$$
\begin{array}{r}
\sigma(\boldsymbol{H})=q_{1} A_{P}^{q_{2}} E^{q_{3}} e^{-\left[|\Delta Z| /\left(q_{5} E^{q_{6}}\right)\right]} \text { for }|\Delta \boldsymbol{Z}| \leq q_{7} \\
=q_{1} A_{P}^{q_{2}} E^{q_{3}} e^{-\left[q_{7} /\left(q_{5} E^{q_{6}}\right)\right]} e^{-\left[\left(|\Delta Z|-q_{7}\right) /\left(q_{8} E^{q_{6}}\right)\right]} \\
\text { for }|\Delta Z|>q_{7}
\end{array}
$$

Best-fit values are $q_{1}=15.53 \pm 1.92 ; \quad q_{2} \pm 0.51 \pm 0.03$; $q_{3}=-1.28 \pm 0.03 ; \quad q_{5}=6.87 \pm 0.11 ; \quad q_{6}=1.43 \pm 0.03$; $q_{7}=7.91 \pm 0.16$; and $q_{8}=4.15 \pm 0.07$. This equation has a $\chi^{2}$ of 2.07 and is our preferred fitting equation, because it dispenses with the complication of an odd-even effect without appreciably increasing $\chi^{2}$. Figure 15 shows an example of the fit to the data from the $\mathrm{Au}$ at 766 $\mathrm{MeV} /$ nucleon runs. Figure 16 shows the distribution of the normalized residuals between the values calculated from Eq. (6) and the data points. Here again, the distri-

TABLE III. Double exponential fits to the hydrogen target data for all the projectiles and energies used in this study.

\begin{tabular}{lcccccc}
\hline \hline & $\begin{array}{c}\text { Beam } \\
\text { mass }\end{array}$ & $\begin{array}{c}\text { Energy } \\
(\mathrm{MeV} / \text { nucleon })\end{array}$ & $\sigma(\mathrm{mb})$ & $\delta$ & $\delta^{\prime}$ & Reduced $\chi^{2}$ \\
\hline $\mathrm{Xe}$ & 131 & 1182 & $171.3 \pm 8.1$ & $7.51 \pm 0.49$ & $5.50 \pm 0.27$ & 1.56 \\
$\mathrm{La}$ & 139 & 516 & $461.2 \pm 20.2$ & $2.64 \pm 0.08$ & $1.95 \pm 0.29$ & 1.86 \\
$\mathrm{La}$ & 139 & 621 & $352.6 \pm 13.5$ & $3.31 \pm 0.10$ & $2.37 \pm 0.20$ & 0.33 \\
$\mathrm{La}$ & 139 & 776 & $248.5 \pm 8.9$ & $5.08 \pm 0.19$ & $2.57 \pm 0.13$ & 1.92 \\
$\mathrm{La}$ & 139 & 906 & $197.5 \pm 8.0$ & $6.64 \pm 0.35$ & $3.09 \pm 0.14$ & 2.00 \\
$\mathrm{La}$ & 139 & 1065 & $161.0 \pm 8.7$ & $7.78 \pm 0.62$ & $4.78 \pm 0.25$ & 1.32 \\
$\mathrm{La}$ & 139 & 1166 & $146.7 \pm 6.7$ & $9.34 \pm 0.73$ & $4.71 \pm 0.20$ & 2.24 \\
$\mathrm{Ho}$ & 165 & 488 & $492.3 \pm 20.8$ & $2.61 \pm 0.08$ & $1.90 \pm 0.26$ & 1.52 \\
$\mathrm{Ho}$ & 165 & 772 & $283.6 \pm 15.5$ & $4.97 \pm 0.25$ & $3.19 \pm 0.16$ & 1.42 \\
$\mathrm{Ho}$ & 165 & 922 & $221.0 \pm 11.3$ & $6.96 \pm 0.44$ & $3.73 \pm 0.15$ & 1.35 \\
$\mathrm{Ho}$ & 165 & 1057 & $180.1 \pm 11.3$ & $7.90 \pm 0.67$ & $5.22 \pm 0.23$ & 0.94 \\
$\mathrm{Au}$ & 197 & 557 & $578.5 \pm 29.4$ & $2.59 \pm 0.08$ & $2.37 \pm 0.22$ & 2.86 \\
$\mathrm{Au}$ & 197 & 665 & $423.5 \pm 20.5$ & $3.47 \pm 0.12$ & $2.30 \pm 0.15$ & 1.42 \\
$\mathrm{Au}$ & 197 & 766 & $325.7 \pm 15.2$ & $4.70 \pm 0.20$ & $2.77 \pm 0.13$ & 2.22 \\
$\mathrm{Au}$ & 197 & 914 & $253.1 \pm 12.1$ & $6.06 \pm 0.32$ & $3.87 \pm 0.14$ & 1.71 \\
$\mathrm{Au}$ & 197 & 972 & $233.4 \pm 12.1$ & $6.37 \pm 0.37$ & $4.39 \pm 0.16$ & 0.98 \\
\hline \hline
\end{tabular}




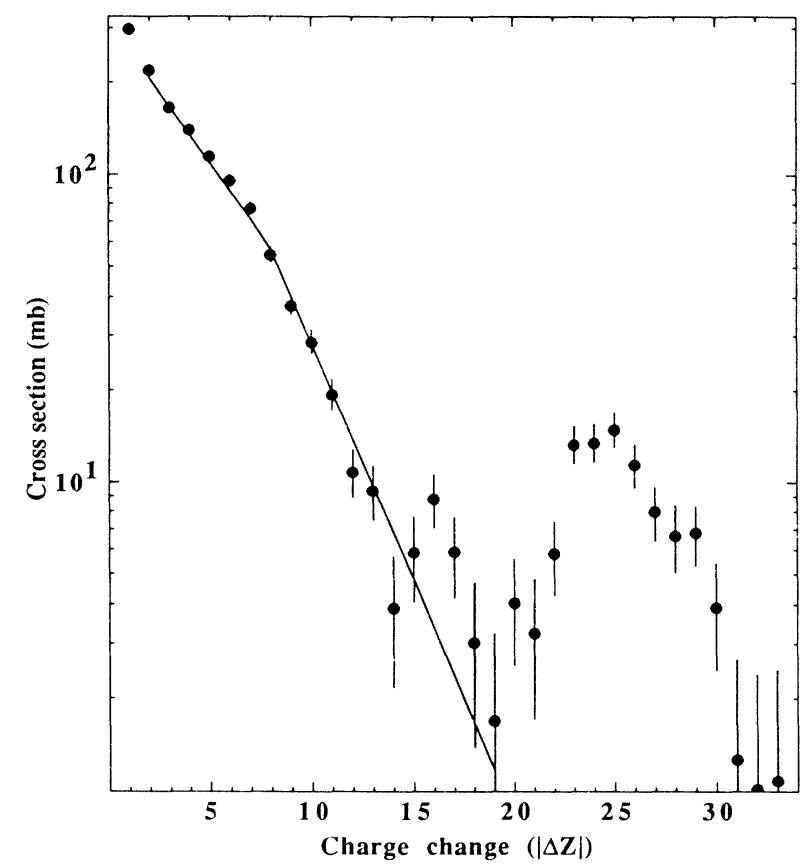

FIG. 15. Fit of Eq. (6) to the measured cross sections of $\mathrm{Au}$ on $\mathrm{H}$ at $766 \mathrm{MeV} /$ nucleon, plotted as a function of charge change $\Delta Z$. The fission peak can be clearly seen.

bution is well characterized by a Gaussian peak, not fitted to all the data but to 232 of the 303 values, with a standard deviation on the plotted curve of 0.10 . In this case there are appreciable tails on either side of the peak, suggesting that some cross sections are not well fitted, and the rms deviation of the entire distribution of the

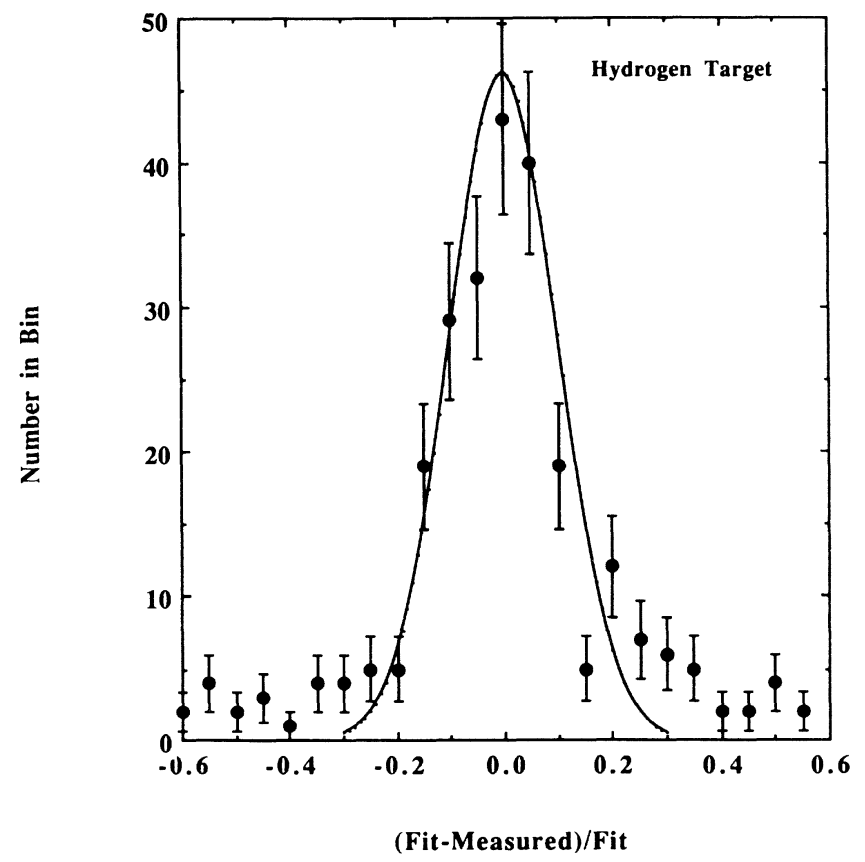

FIG. 16. Normalized residuals, formed from the difference between the calculated and measured values of cross sections from Eq. (6) divided by the calculated values. A Gaussian function with an area of 232 of the 303 values and with a standard deviation of 0.10 is also shown. data is 0.9 . However, many of the cross sections with large $|\Delta Z|$ are quite small and have considerable fractional uncertainties. It is these that are largely responsible for these apparent bad fits. This is illustrated in Fig. 17 (a), which shows the distribution of these residuals relative to the uncertainty on each measured value. For

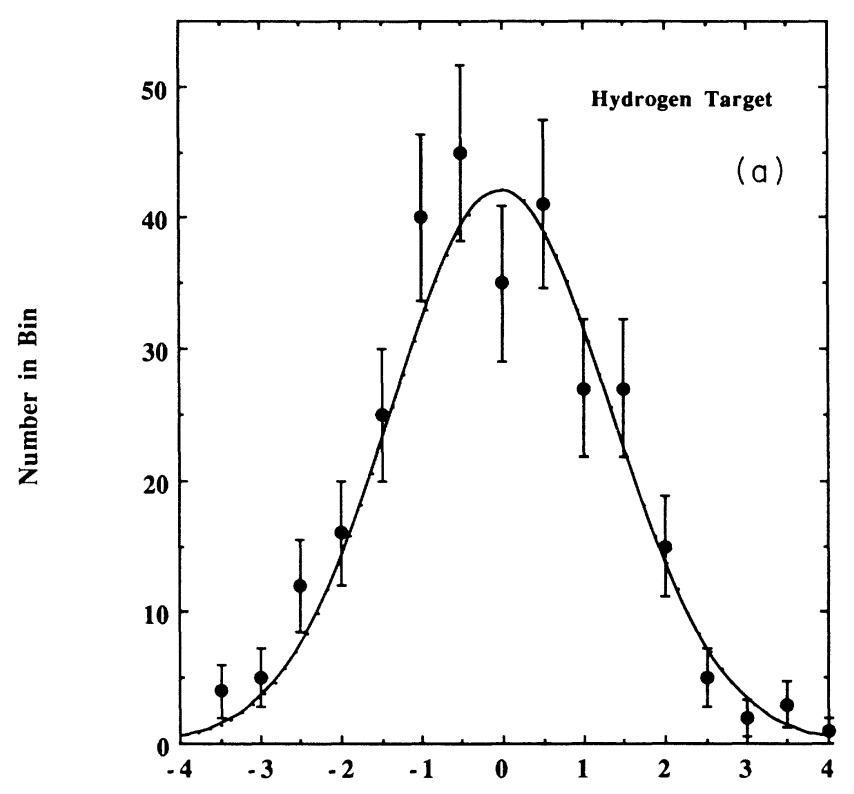

(Fit-Measured)/Uncertainty

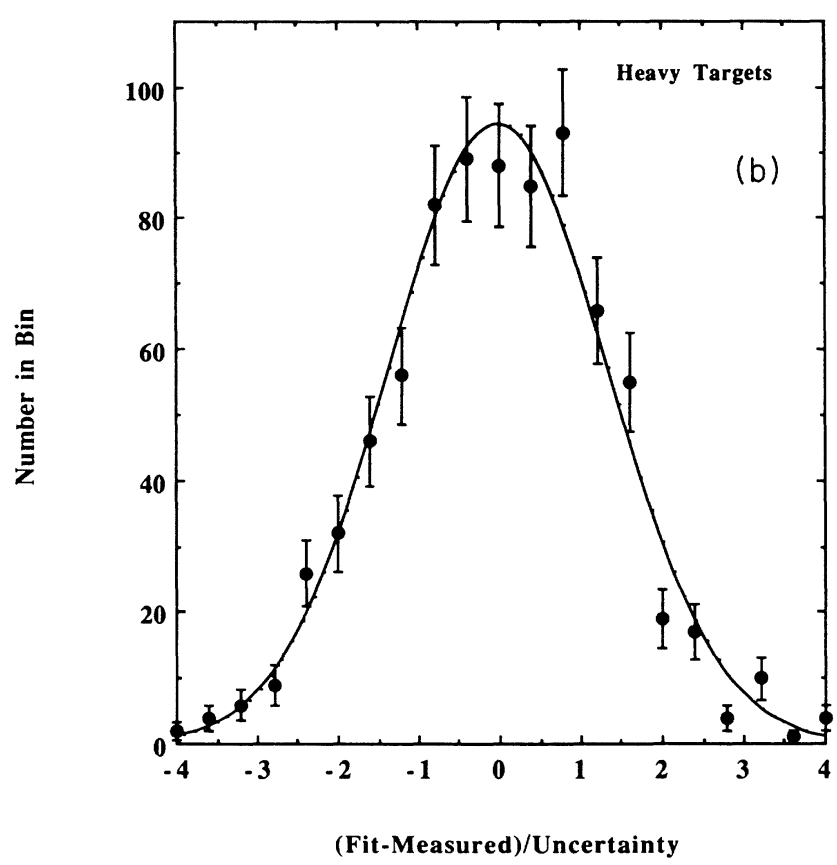

FIG. 17. (a) Ratio of the residuals between the calculated and measured values in hydrogen from Eq. (6) to the experimental uncertainties in the measured cross sections. A Gaussian function of equal area to the data with $\sigma=1.3$ is also shown. (b) Ratio of the residuals between the calculated and measured values in heavy targets from Eq. (4) to the experimental uncertainties in the measured cross sections. A Gaussian function of equal area to the data with $\sigma=1.3$ is also shown. 
comparison Fig. 17(b) shows a similar plot for the heavy target fits, indicating that when the uncertainties are taken into account both sets of fits are very similar, each having standard deviations of 1.3. Alternatively, we can examine only those cross sections with small uncertainties. Thus, for $2 \leq|\Delta Z| \leq 10$, the rms deviation of the complete normalized residual distribution is only 0.096 , while for $2 \leq|\Delta Z| \leq 15$, the rms deviation is still only 0.25 and $95 \%$ of those cross sections are within a factor of 1.50 of the fit. The cross sections with $|\Delta Z|>15$ do not contribute disproportionately to $\chi^{2}$, because of their small magnitude and large uncertainties, hence the great variation in the ratios does not necessarily reflect the goodness of the fit for large $|\Delta Z|$. No regular residual dependences are evident.

The odd-even factor, which is a simplistic expression of the effect of nuclear structure, does not appear to be needed. The Xe projectiles are the only ones with a clear odd-even variation for the first few charge changes. Even this projectile shows a contrary effect at $\Delta Z=-8$.

Given the large $\chi^{2}$ s obtained by fitting even a double exponential equation to each run separately (see Table III), it is unlikely that the overall fit can be markedly improved with the available data, since the separate fits yield $\chi^{2}$ s of similar size to the overall $\chi^{2}$. It is particularly important to obtain data with a larger variation in the projectile mass, in order to confirm the lack of dependence on projectile mass of the $\delta$ and $\delta^{\prime}$ parameters, and with a greater number of even charged projectiles, so that the odd-even effect of the fragmentation can be further studied for these nuclei. In addition, the break in the slope of the exponential may or may not be related to the fact that the odd-even effect applies only to small $\Delta Z$ 's. The two are explicitly connected in the overall fitting equation, the break of both occurring at a value between $\Delta Z=-7$ and $\Delta Z=-8$, with the exact value being unimportant to the goodness of fit. The odd-even effect is not visible in the heavy target cross sections (except in the case of iron; see below). Perhaps these results indicate that an interaction which disturbs the projectile nucleus only slightly is strongly affected by pairing of protons.

\section{E. Comparison with semiempirical values}

We have used the semiempirical relations proposed by Silberberg et al. ${ }^{12}$ to calculate values for the cross sections at the nominal energies of our runs. Comparisons between our fitted values at $1000 \mathrm{MeV} /$ nucleon and those predicted by the semiempirical relations are shown in Fig. 18. It can be seen that there is a strong dependence on the projectile mass, with the Au semiempirical predictions ranging from being more than $50 \%$ above to almost a factor of two below our fit predictions and the lighterprojectile nuclei being mostly some $50 \%$ above them. The semempirical values between $2 \leq \Delta Z \leq 20$ have also been compared with the measured values in Fig. 19, which shows the normalized residuals, and can be compared with the similar comparison to our fitted values, Fig. 16. The superiority of our fits over the semiempirical fits is clearly illustrated by this comparison.

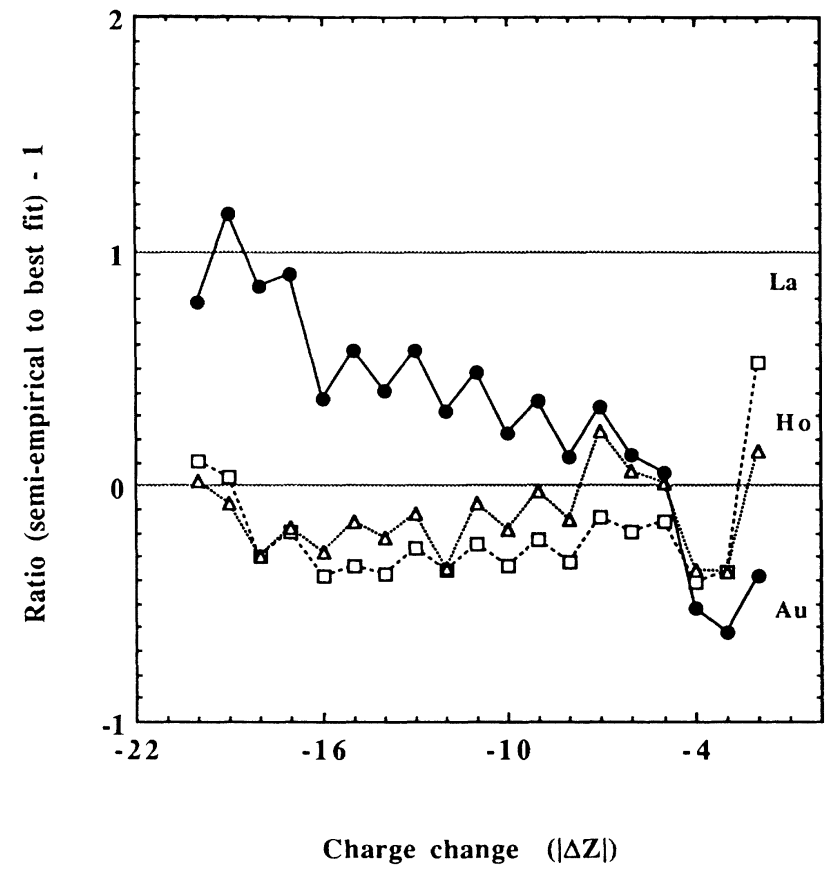

FIG. 18. Comparison between the semiempirical predictions and those from Eq. (6) for $\mathrm{Au}, \mathrm{Ho}$, and $\mathrm{La}$ at 1000 $\mathrm{MeV} /$ nucleon.

\section{SPECIAL CROSS SECTIONS}

\section{A. Charge change of minus one}

As stated above, the cross sections for fragments with $\Delta Z=-1$ are systematically larger than the general trend of the cross sections for $|\Delta Z| \geq 2$ would lead us to expect. The physical significance of the empirical fits to the cross sections for $2 \leq|\Delta Z| \leq 20$ are not fully understood.

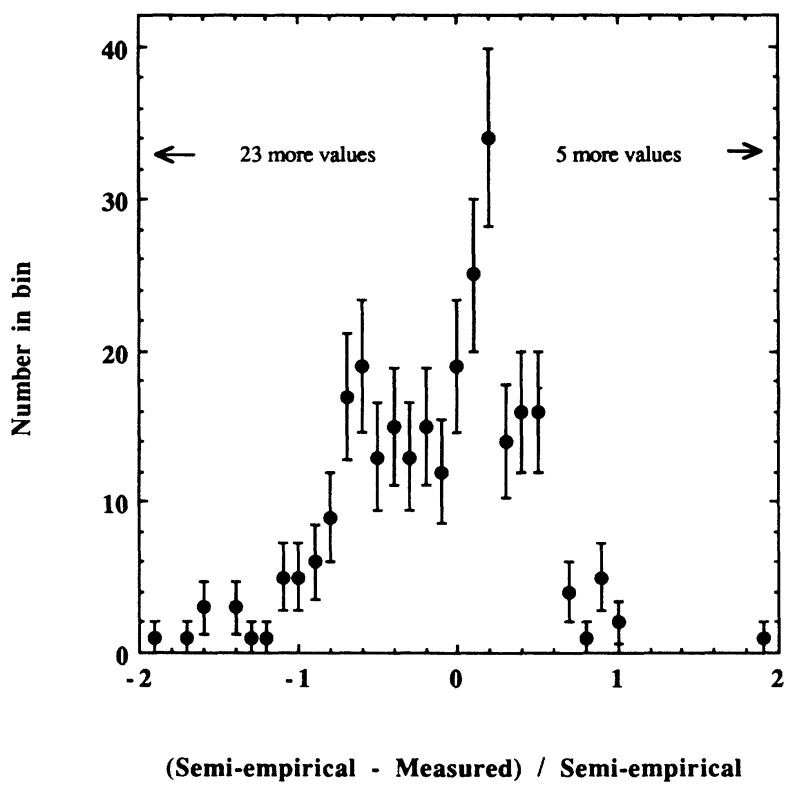

FIG. 19. Normalized residuals between semiempirical and experimentally determined cross sections in hydrogen for 191 of the 228 values. Compare with Fig. 16. 
Hence, one cannot necessarily expect that the $\Delta Z=-1$ cross sections will follow the predictions of these fits. But at least the power-law relationship for the heavy targets is so well obeyed over such a broad range of targets, projectiles, and energies that it reasonable to suppose that it does describe the general behavior of peripheral nuclear interactions. It is thus reasonable, even if not strictly justifiable, to use the power-law relationship as an empirical fact, and to compute the excess $\Delta Z=-1$ cross section, while observing that this excess could be due to the operation of a different, and additional, interaction mechanism(s), or merely an empirical fact that is useful to complete the global fit.

The mechanism of electromagnetic dissociation (EMD) can readily cause the loss of one charge, and at sufficiently high energies, several charges. ${ }^{25}$ Relativistic nuclei passing a target nucleus at a small impact parameter, but one large enough to avoid nuclear interaction, experience an electromagnetic pulse from the Lorentz contracted field of the target. The excitation of a multipole resonance in the projectile nucleus from the exchange of a virtual photon results in the emission of one or more nucleons. Neutron emission is preferred because of the Coulomb barrier against proton emission, however the latter does occur, particularly, one would expect, in those nuclei with smaller charges. Cross sections for the stripping of one neutron from a target gold nucleus by a $\mathrm{La}$ projectile of Bevalac energy are as large as $2 b^{26,27}$

Other researchers have found that at very high energies, beyond those available at the Bevalac, the cross sections for presumed EMD become many times the nuclear cross sections, especially for heavy targets and nuclei. ${ }^{28,24}$ They also find a large dependence for the neutron stripping reaction even at Bevalac energies on projectile and target charge. Proton stripping requires surmounting the Coulomb barrier, which is higher for heavy nuclei, countering the greater degree of excitation in highcharged nuclei, although this does not explain the lack of target dependence. In any case, we can still attempt empirical fits.

Figure 20 illustrates the 58 excess cross sections $\left(\sigma_{\text {exc }}\right)$ for $\Delta Z=-1$, derived from the excess observed over the power law or exponential fit prediction from each separate run to those cross sections for $2 \leq|\Delta Z| \leq 20$ for heavy projectiles on all targets. We did not use the global fit to determine the excesses because for the few runs which were relatively poorly represented by the global equation, an artifact would be present in the excess. The scatter in the values of $\sigma_{\text {exc }}$ is large, with a few values even being negative. The gold values show a particularly large variation with energy. At low energies $\sigma_{\text {exc }}$ is very large, comparable to the presumed nuclear cross sections. At high energies, the values converge to a constant value of about $40 \mathrm{mb}$ not dependent on projectile or target and comparable to the values found for the $\Delta Z=+1$ cross sections. This lack of variation with target charge is not what one would expect for an electromagnetic process, which should be strongly charge dependent, suggesting that possibly the mechanism producing the excess minus one cross sections is not solely EMD.

We have attempted to find an equation which could fit

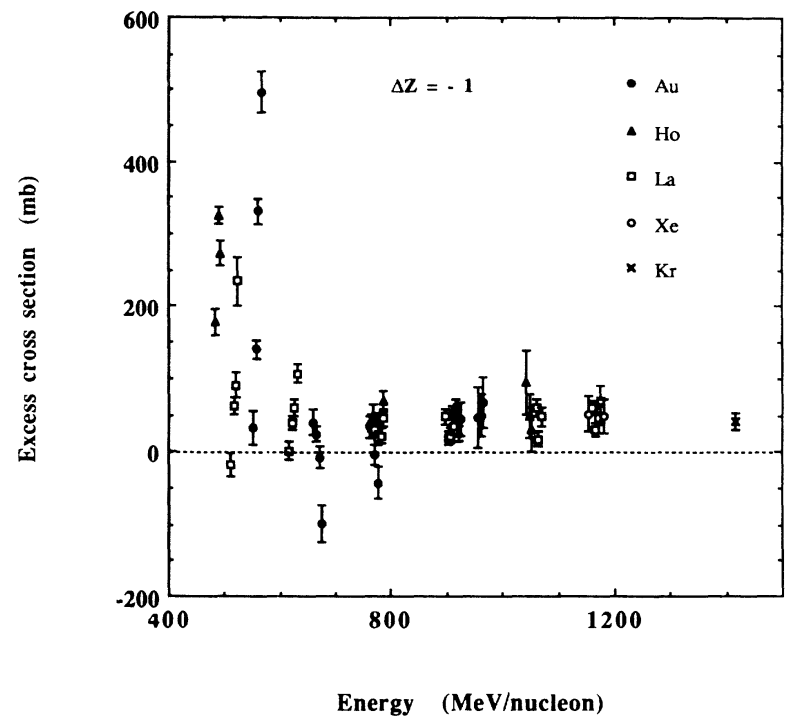

FIG. 20. The excess cross sections for $\Delta Z=-1$ observed in all targets and for all projectiles, as a function of energy. For clarity, results at nominally similar energies are displaced to the right in energy on heavy targets with respect to those on lighter targets.

the values of $\sigma_{\text {exc }}$, but the incoherent nature of the measurements made this difficult. We were forced to conclude that more data is needed to explore the low-energy region and the effect of using even charged projectile nuclei before a reliable equation can be found. For the cross sections of projectile nuclei above $700 \mathrm{MeV} /$ nucleon, the excess is well described by a constant value of $42 \pm 22 \mathrm{mb}$. This excess can be used in combination with the global fits above to find cross sections in this energy regime.

\section{B. Cross sections for charge pickup, $\Delta Z=+1$}

These have been discussed in detail in the companion paper, ${ }^{1}$ but there it was shown that again there was no simple global fit to all the data on these cross sections.

\section{Large charge changes}

The trend of Eqs. (1) and (2) cannot continue out to very large values of $|\Delta Z|$ because the values of the partial cross sections would not add up to the total cross sections for charge change. Unfortunately, for $\Delta Z \gg 20$, where the cross sections are quite small, there are large statistical uncertainties. In addition, the fragments other than the largest begin to obscure the peaks of the charge of the leading fragment, and the resolution deteriorates. Still, except in the case of gold, which fissions, clear peaks are usually discernible in the total signal histograms to $\Delta Z$ beyond -30 . Even for gold, where we have the additional confusion of the occurrence of fission, considered in the next section, one can see charge peaks beyond the fission region from $\Delta Z \approx-30$ to -38 .

The cross section excesses above the predictions of the equations for La projectiles, are shown in Fig. 21. Similar excesses are seen for Ho projectiles. The power-law and exponential fits remain close to the measured values 
up to $|\Delta Z| \approx 26$ for both $L$ and $H$. Above these values, at least in the higher-energy runs, these excesses begin to increase; i.e., the measured cross sections tend to a constant value. Presumably a different interaction mechanism dominates in this region. Silberberg et al. ${ }^{12}$ refer to peripheral and spallation interactions which change over for fragments with a charge of less than about $40 \%$ of the projectile charge to breakup and fission interactions.
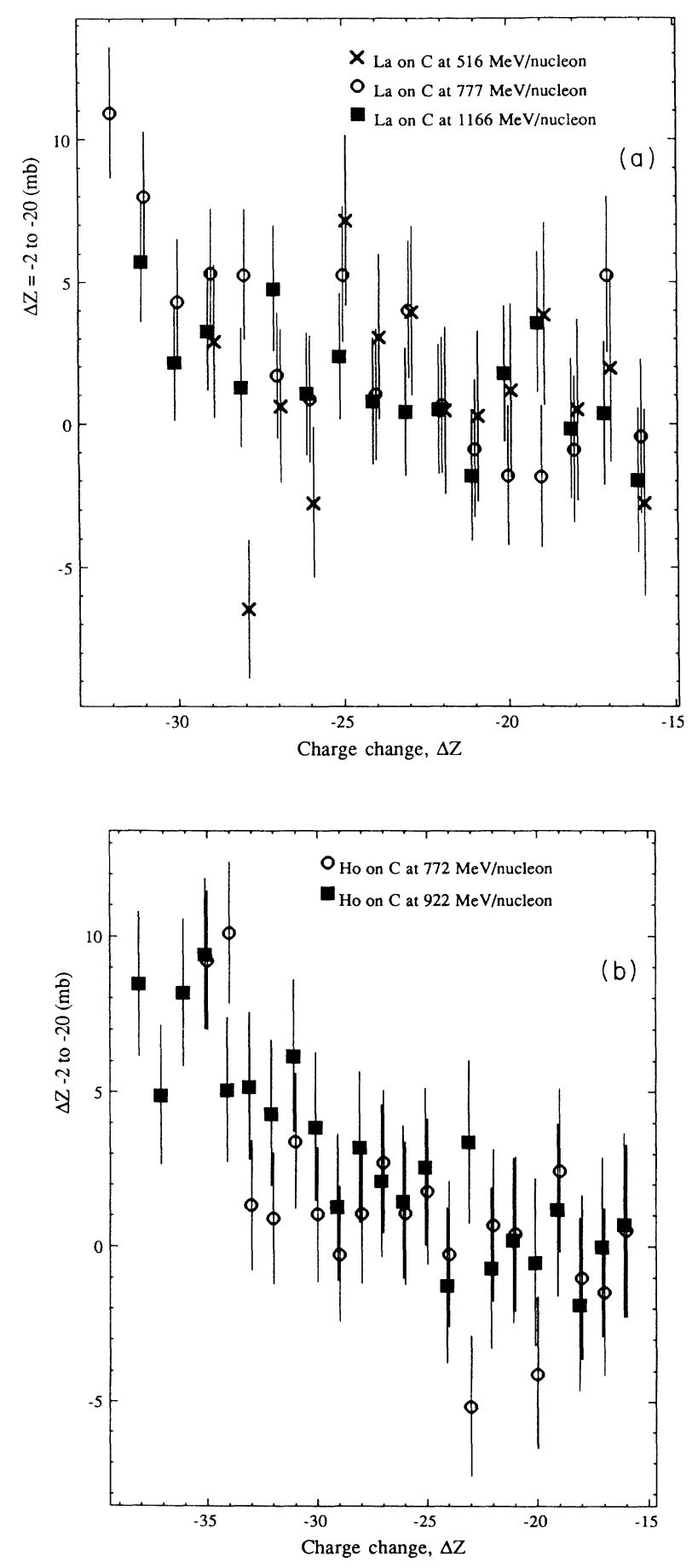

FIG. 21. The excess cross sections above the predictions of fit for large $|\Delta Z|$ for $\mathrm{La}$ on $\mathrm{C}$ at several energies.

\section{Fission of gold}

Gold has a significant probability of experiencing induced fission due to interactions at Bevalac energies. ${ }^{29}$ Fragments due to symmetric fission produce a signal in our detector that is about the same as the signals of a single $Z=56$ fragment, $|\Delta Z|=23$, while slightly asymmetric fission and fission with the loss of a few single charges produces somewhat lower signals, corresponding to larger $\Delta Z$ 's. These fission peaks are not clearly seen on logarithmically compressed plots of the cross sections except for the lightest targets. Figure 22 shows linear plots for $\mathrm{Au}$ on $\mathrm{C}, \mathrm{Al}$, and $\mathrm{Au}$, and clearly illustrates a fission peak for each target and each energy. It was impossible on an event-by-event basis to distinguish fission from fragmentation in this analysis, so the cross sections measured for the charges in this region include the cross section for near symmetric fission. As discussed in the previous section, the cross sections for large $|\Delta Z|$ of lighter nuclei follow the power-law or exponential fits to $Z_{F}$ of about $60 \%$ of $Z_{P}$, depending on the mass of the target. In either case they appear to project well into the fission region on the charge scale. We have assumed that they are valid throughout the range of $\Delta Z$ for which fission events would appear. Since the observed cross sections at large $\Delta Z$ tend to be greater than those predicted, Sec. IV C, this procedure should tend to overestimate the fission cross sections.

By subtracting the power-law fit or double exponential fit to separate runs from the measured cross sections in the region near $\Delta Z=-23$ and assuming a Gaussian distribution in the fission peak region, we calculated cross sections for fission from our data. Table IV contains the cross sections derived here, and also the cross sections reported from an earlier experiment, ${ }^{30}$ which did not measure cross sections for very large $\Delta Z$ 's, those beyond the fission peak, and had errors of the order of $20 \%$. The errors quoted on the cross sections obtained here do not reflect the uncertainties of the subtraction, which should bias the results to lower values.

Vaishnene et al. ${ }^{31}$ measured a cross section of $71 \pm 7$ $\mathrm{mb}$ for the fission of gold by proton projectiles at $1 \mathrm{GeV}$. Our values are close to this for our gold projectiles on a hydrogen target. Appreciably larger cross sections have been reported for energetic proton and ${ }^{14} \mathrm{~N}$ projectiles ${ }^{32,33}$ on gold. There seems to be no way to accommodate such large fission cross sections within our data, since in some cases they even exceed the total observed cross section in this range of $\Delta Z$.

There is very little energy dependence, but the shape of the fission peak is displaced slightly toward smaller charge changes, implying more symmetric fission, for lower energies. However, this could be an effect of the higher velocity in the Cherenkov detector of the fission fragments relative to the projectile nuclei or the fragments with comparable signal. Since we determined the factors for combination of the ion counter and Cherenkov signals to remove energy dependence of the total signal from smaller $|\Delta Z|$ 's, the total signals in this $\Delta Z$ region could be energy dependent. 
TABLE IV. Derived cross sections for fission of Au projectiles. Errors are nominal statistical errors only and do not reflect a probable overestimate due to subtraction of predicted single fragment cross sections.

\begin{tabular}{lcc}
\hline \hline Target & $\begin{array}{c}\text { Energy } \\
(\mathrm{MeV} / \text { nucleon })\end{array}$ & $\begin{array}{c}\text { Observed fission } \\
\text { cross section }(\mathrm{mb})\end{array}$ \\
\hline $\mathrm{H}$ & 557 & $68.3 \pm 5.0$ \\
$\mathrm{H}$ & 665 & $82.4 \pm 5.1$ \\
$\mathrm{H}$ & 766 & $89.6 \pm 5.6$ \\
$\mathrm{H}$ & 914 & $69.8 \pm 5.4$ \\
$\mathrm{C}$ & & \\
$\mathrm{C}$ & 557 & $67.0 \pm 7.8$ \\
$\mathrm{C}$ & 666 & $44.4 \pm 7.3$ \\
$\mathrm{C}$ & 766 & $46.0 \pm 8.2$ \\
& 915 & $66.9 \pm 7.2$ \\
$\mathrm{Al}$ & & \\
$\mathrm{Al}$ & 557 & $75.3 \pm 8.4$ \\
$\mathrm{Al}$ & 666 & $59.4 \pm 8.0$ \\
$\mathrm{Al}$ & 766 & $75.3 \pm 8.7$ \\
$\mathrm{Cu}$ & 915 & $63.4 \pm 8.2$ \\
$\mathrm{Cu}$ & & \\
$\mathrm{Cu}$ & 559 & $94.8 \pm 9.7$ \\
$\mathrm{Cu}$ & 667 & $94.8 \pm 9.5$ \\
& 767 & $76.4 \pm 10.3$ \\
$\mathrm{H}$ & 915 & $85.3 \pm 10.3$ \\
$\mathrm{C}$ & & \\
$\mathrm{Al}$ & Kertzman, Ref. 30 & 63 \\
\hline
\end{tabular}

\section{E. Iron-projectile cross sections}

We collected data from runs with an iron projectile on carbon, aluminum, copper, and lead targets at one energy, near the maximum rigidity at the Bevalac, about 1550 $\mathrm{MeV} /$ nucleon in the center of the target. Although cross sections for small $\Delta Z$ of iron-projectile nuclei follow roughly a power law in $\Delta Z$, there is a strong odd-even effect $\approx 20 \%$ and an enhancement in the cross section for production of silicon $(Z=14)$. Cross sections for $\Delta Z \approx-9$ to the limit of our sensitivity, about $\Delta Z \approx-16$, are roughly constant. There is very little variation in the shape of the elemental cross section distribution with the target, which supports the applicability of factorization to this data. We fit a power law in the $\Delta Z$ equation, including an odd-even factor proportional to $\epsilon$, to each of the runs separately:

$$
\sigma(\mathrm{Fe})=\sigma_{\mathrm{Fe}}\left[1+\epsilon(-1)^{Z_{F}}\right]|\Delta Z|^{-\beta^{\prime}} .
$$

For $2 \leq|\Delta Z| \leq 8$, this equation has reduced $\chi^{2}$,s of about 2. For larger $\Delta Z$ 's, a constant in $\Delta Z$ times an odd-even factor fits with $\chi^{2}$ 's of about 3 . The cross section for the production of silicon, which shows a large increase, deviates most from this equation.

\section{CONCLUSIONS}

A complication arises when we turn to the question of the application of these results to the propagation of nuclei in space. This experiment was designed to find charge-changing interaction cross sections. But in the
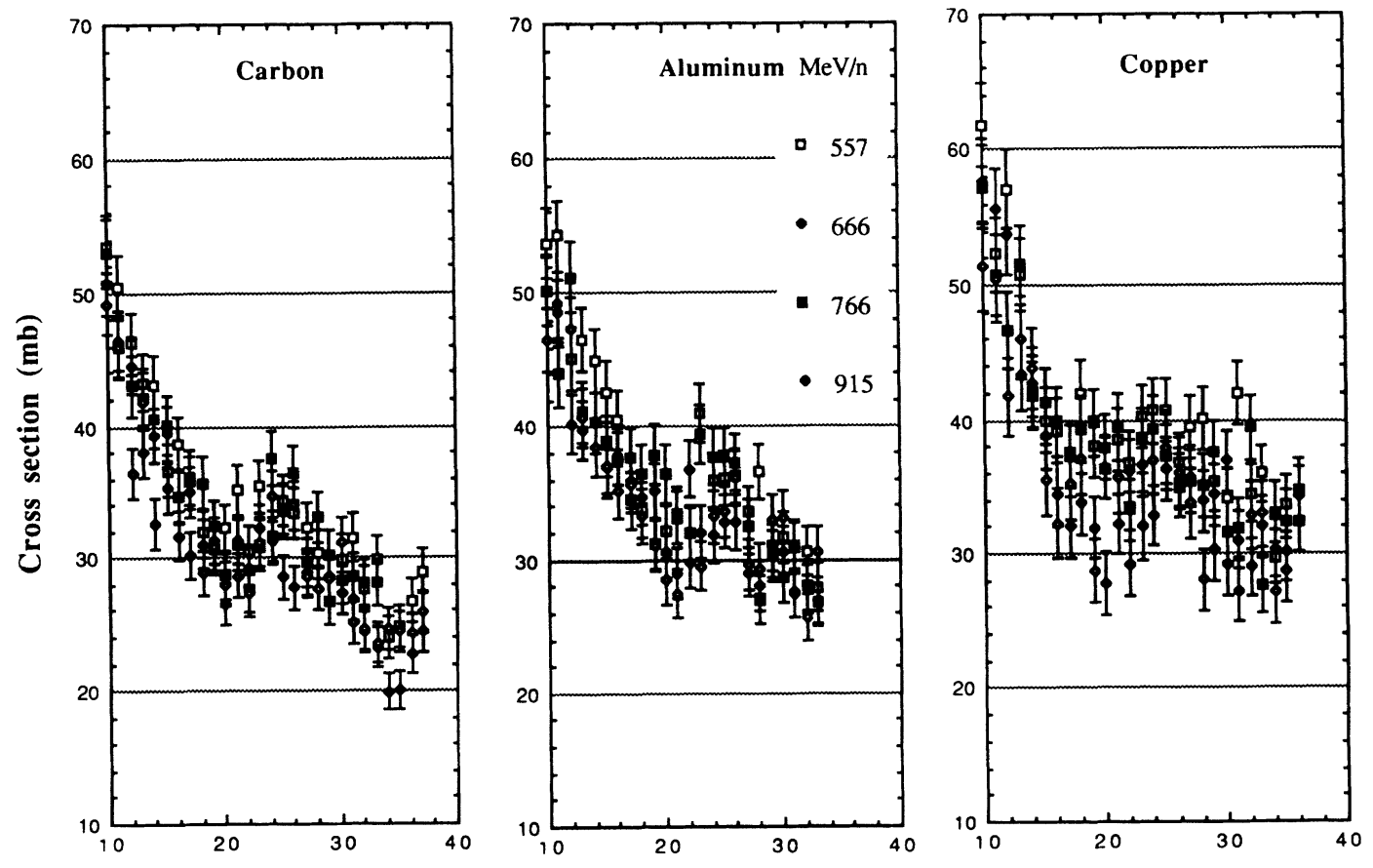

Charge change $(\Delta \mathrm{Z})$

FIG. 22. Cross sections, in mb, of Au at all energies on all three heavy targets for those fragments with $\Delta Z \geq 10$ plotted on linear scales as a function of $\Delta Z$. Increases attributable to fission can be seen in every case. 
propagation problem, where there exists sufficient time for the decay of radioactive nuclei with $\tau \leq 10^{6}$ years, we need the isotopic cross sections in order to determine the production of elements after allowing for decay. Our results ${ }^{1}$ give us some indication of the isotopic production from the measures of the neutron losses seen for $|\Delta Z| \leq 10$, but they cannot give the individual isotopic yields. This limitation will be unavoidable until there have been considerable improvements in detectors. For the application of the results of this experiment to the interaction of particles in the material surrounding a cosmic ray detector, these isotopic considerations are not important, since decays do not have time to occur.

Although our global fits only allow us to predict cross sections over a limited range of $\Delta Z$, this should be adequate for most cosmic ray propagation problems. The abundance of elements increases rapidly as $Z$ decreases. Hence the contribution of fragments with large $\Delta Z$ to the overall abundances is generally overwhelmed by the natural abundance of the elements with that $Z$. Only in the region of the lanthanides $Z \geq 57$ is there likely to be a problem, since the only major peak in the abundances of the heavier elements in the cosmic ray source is probably in the $\mathrm{Pt}-\mathrm{Pb}, 76 \leq Z \leq 82$, region. Even here, the cross sections for such large values of $\Delta Z$ are so small that the contribution of $\mathrm{Pt}-\mathrm{Pb}$ fragments to the observed abundances at $Z \approx 60$ will be small.

We have been able to generate a seven-parameter global fit to all 795 measured cross sections with $2 \leq \Delta Z \leq 20$ for the heavy targets which fits the data with a standard deviation of $7 \%$. We have also generated a similar global fit to 303 measured cross sections for a hydrogen target which fits $77 \%$ of the data with a standard deviation of $10 \%$. These global fits are the best available at this time and will be improved only when further data has been obtained for additional targets, projectiles, and energies. In making these fits we have found better results by not using weak factorization or the hypothesis of limiting fragmentation. We plan to continue these studies and expect to obtain still better representations of the variations of the cross sections with the various relevant parameters in the future.

This study was partially supported by NASA Grants NAG 8-498, -500, -502 and NGR 05-002-160, 24-005-050 and 26-008-001.
*Present address: California Institute of Technology, Pasadena, CA 91125.

1J. R. Cummings, T. L. Garrard, M. H. Israel, J. Klarmann, E. C. Stone, C. J. Waddington, and W. R. Binns, Phys Rev. C 42, 2508 (1990), the preceding paper.

${ }^{2}$ W. R. Binns, T. L. Garrard, P. S. Gibner, M. H. Israel, M. P. Kertzman, J. Klarmann, B. J. Newport, E. C. Stone, and C. J. Waddington, Astrophys. J. 346, 997 (1989).

${ }^{3}$ J. P. Meyer, Proceedings of the 19th International Cosmic Ray Conference, La Jolla, California (NASA Conf. Pub. No. 2376, 1985), Vol. 2, p. 267 (1985).

${ }^{4} \mathrm{M}$. H. Israel, Proceedings of the 12th Texas Symposium on the Relativity of Astrophysics, edited by M. Livio and G. Shaviv [Ann. N.Y. Acad. Sci. 470, 188 (1986)].

${ }^{5}$ W. R. Binns, T. L. Garrard, M. H. Israel, M. P. Kertzman, J. Klarmann, E. C. Stone, and C. J. Waddington, Phys. Rev. C 36, 1870 (1987).

${ }^{6}$ W. R. Binns, J. R. Cummings, T. L. Garrard, M. H. Israel, J. Klarmann, E. C. Stone, and C. J. Waddington, Phys. Rev. C 39, 1785 (1989).

${ }^{7}$ J. B. Cumming, P. E. Haustein, J. J. Ruth, and G. J. Virtes, Phys. Rev. C 17, 1632 (1978).

${ }^{8}$ D. J. Morrissey, W. Loveland, and G. T. Seaborg, Z. Phys. A 289, 2293 (1979).

${ }^{9}$ N. T. Porile, G. D. Cole, and C. R. Rudy, Phys. Rev. C 19, 2288 (1979).

${ }^{10}$ See the review by H. Boggild and T. Ferbel, Annu. Rev. Nucl. Sci. 24, 451 (1974).

${ }^{11}$ More precisely, if the momenta of the target and projectile nuclei are $p_{T}$ and $p_{P}$ then when $s=\left(p_{T}+p_{P}\right)^{2}$ cross sections tend to limiting values as $s$ becomes large.

${ }^{12}$ R. Silberberg, C. H. Tsao, and J. R. Letaw, Proceedings of the 20th International Cosmic Ray Conference, Moscow, 1987, Vol. 2, p. 133, and references therein.

${ }^{13}$ A. S. Hirsch, A. Bujak, J. E. Finn, L. J. Gutay, R. W. Minch, N. T. Porile, R. P. Scharenberg, B. C. Stringfellow, and F. Turkot, Phys. Rev. C 29, 508 (1984).
${ }^{14}$ A. D. Panatagiotou, M. W. Curtin, H. Toki, D. K. Scott, and P. J. Siemens, Phys. Rev. Lett. 52, 496 (1984).

${ }^{15} \mathrm{G}$. Rudstam, Z. Naturforsch. 219, 1027 (1966).

${ }^{16}$ J. B. Cumming, P. E. Haustein, R. W. Stoenner, B. Mausner, and R. A. Naumann, Phys. Rev. C 10, 739 (1974).

${ }^{17}$ D. L. Olson, B. L. Berman, D. E. Greiner, H. H. Heckman, P. J. Lindstrom, and H. J. Crawford, Phys. Rev. C 28, 1602 (1983).

${ }^{18}$ P. R. Bevington, Data Reduction and Error Analysis for the Physical Sciences (McGraw-Hill, New York, 1969), Sec. 11-4.

${ }^{19}$ W. R. Frazer, L. Ingber, C. H. Mehta, C. H. Poon, D. Silverman, K. Stowe, P. D. Ting, and H. J. Yesian, Rev. Mod. Phys. 44, 284 (1972).

${ }^{20}$ J. Cugnon and R. Sartor, Phys. Rev. C 21, 2342 (1980).

${ }^{21}$ J. Hufner, Phys. Rep. 125, 129 (1985).

${ }^{22}$ J. Hufner and D. Mukhopadhyay, Phys. Lett. B 173, 373 (1986).

${ }^{23}$ P. J. Karol, Phys. Rev. C 11, 1203 (1975).

${ }^{24}$ J. R. Cummings, Ph.D. thesis, University of Minnesota, 1989.

${ }^{25}$ P. B. Price, Ren Guoxiao, and W. T. Williams, Phys. Rev. Lett. 61, 2193 (1988).

${ }^{26}$ M. T. Mercier, J. C. Hill, F. K. Wohn, C. M. McCullough, M. E. Nieland, J. A. Winger, C. B. Howard, S. Renwick, D. K. Matheis, and A. R. Smith, Phys. Rev. C 33, 1655 (1986).

${ }^{27}$ J. C. Hill, F. K. Wohn, J. A. Winger, M. Khayat, K. Leininger, and A. R. Smith, Phys. Rev. C 38, 1722 (1988).

${ }^{28}$ J. C. Hill, F. K. Wohn, J. A. Winger, and A. R. Smith, Phys. Rev. Lett. 60, 999 (1988).

${ }^{29}$ C. J. Waddington and P. S. Freier, Phys. Rev. C 31, 888 (1985).

${ }^{30}$ M. P. Kertzman, Ph.D. thesis, University of Minnesota, 1987.

${ }^{31}$ L. A. Vaishnene, L. N. Andronenko, G. G. Korshevny, A. A. Kotov, G. E. Solyakin, and W. Neubert, Z. Phys. A 302, 143 (1981).

${ }^{32}$ J. Hudis and S. Katcoff, Phys. Rev. C 13, 1961 (1976).

${ }^{33}$ S. Katcoff and J. Hudis, Phys. Rev. C 14, 628 (1976). 